\title{
Wild edible plants collected by Hani from terraced rice paddy agroecosystem in Honghe Prefecture, Yunnan, China
}

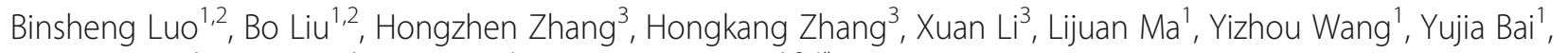
Xinbo Zhang ${ }^{1}$, Jianqin $\mathrm{Li}^{1}$, Jun Yang ${ }^{4}$ and Chunlin Long ${ }^{1,2,4^{*}}$

\begin{abstract}
Background: The Hani people in the Honghe Prefecture of Southeastern Yunnan, China, have practiced terraced rice paddy farming for more than 1300 years. These rice fields, combined with the surrounding forests and water systems, form a special agroecosystem that has attracted both tourists and scientists. For centuries, the local people have traditionally collected wild edible plants (WEP) from the agroecosystem, but this unique traditional practice in this area has never been reported.

Methods: Ethnobotanical fieldwork was conducted in four counties (Yuanyang, Honghe, Jinping, and Lüchun) between 2014 and 2019. Local self-identified Hani people (186) were interviewed, and information concerning local WEP species was obtained, documented, and analyzed. Plant samples and voucher specimens were collected for taxonomic identification.

Results: A total of 224 WEP species, belonging to 90 families and 170 genera, were recorded as used by the Hani people in Honghe. The most common WEP parts used include fruits, stems, and leaves, and the most common preparation methods include eating as a potherb (wild vegetable) and eating fresh. Some WEPs, like Phyllanthus emblica and Dioscorea subcalva, have unique preparation methods. The use-value (UV) and frequency of utilization index (FUI) of WEP species were analyzed. The 20 WEP species with the highest UV were noted as particularly important to the Hani people's daily life in Honghe.

Conclusion: A large majority of these WEP species possess tremendous economic potential for future development. However, the diversity of WEP species, the associated traditional knowledge, and the broader agroecosystem are facing challenges such as biodiversity loss and pollution from chemical pesticides and fertilizers. This study may help local people to recognize the value of local WEP species and associated traditional knowledge, as well as provide ethnobotanical information for the future development of this tourism region.
\end{abstract}

Keywords: Hani terraced rice paddy fields, Wild edible plants, Ethnobotany, Hani ethnic group

\section{Background}

The terraced rice paddy fields of the Hani people of Southeastern Yunnan, China, represent a unique agroecosystem with significant economic, ecological, and esthetic values [1]. Due to the dramatic altitudinal range in this area (144-2939 $\mathrm{m})$ [2], there is a significant diversity

\footnotetext{
*Correspondence: long@mail.kib.ac.cn; long.chunlin@muc.edu.cn

${ }^{1}$ College of Life and Environmental Sciences, Minzu University of China, Beijing 100081, China

${ }^{2}$ Key Laboratory of Ethnomedicine (Minzu University of China), Ministry of Education, Beijing 100081, China

Full list of author information is available at the end of the article
}

of climatic zones and associated micro-climates [3]. These climatic zones, in order from low to high altitude, are southern subtropical, middle subtropical, northern subtropical, warm temperate, temperate, and cold temperate climates [3]. This complex topography and diversity of climates significantly contribute to the richness of local biodiversity [4].

Since the 1960s, the Hani terraced rice paddy fields have attracted scientific interest, and they have even been elected into the Globally Important Agricultural Heritage Systems (2010) and the UNESCO World Heritage List

(c) The Author(s). 2019 Open Access This article is distributed under the terms of the Creative Commons Attribution 4.0 International License (http://creativecommons.org/licenses/by/4.0/), which permits unrestricted use, distribution, and 
(2013) [5]. For example, Zhu et al. [6] carried out a series of experiments in the terraced rice paddy fields in Honghe Hani and found that crop heterogeneity could solve the vulnerability of monoculture crops to disease; Li et al. [7] studied the agricultural soils by molecular methods and revealed the dynamics of organic matter in Yuanyang Terrace. However, there have been no studies on the wild edible plants (WEP species) collected and consumed by the Hani people in Honghe Hani terraced rice paddy system. In addition to scientific interest, the Honghe rice terraces have attracted more than 20 million tourists since 2014 [8]. Due to their interests in local foods, tourists have driven a demand for WEP species on the menus of local restaurants.

The Hani people speak their own language, which does not have a traditional writing system. After 1957, a set of writing characters of the Hani language based on Latin was invented with the help of the Chinese government and linguists [8].

About 1300 years ago [9], the Hani people migrated to Southeast Yunnan and began the cultivation of rice paddies in terraced hillside fields, forming a sustainable agroecosystem consisting of four major components: forests, villages, terraced rice paddies, and river systems (Fig. 1) [10, 11]. The evergreen forests control the water in the soil, acting like a natural reservoir to maintain the water year long, and also provide water for the villages and terraced rice paddies in lowlands through water channels built by Hani people [10]. Also, the hot and humid valley climate frequently generates a thick fog that helps to maintain moist air throughout the year. This unique four element-based Hani agroecosystem ensures a stable water supply. Consequently, the Hani terraced rice paddy fields did not suffer any significant damage from the historic 2009-2010 drought in China $[2,12]$.

Due to this unique and complex agroecosystem, together with its rich biodiversity, much traditional ethnobotanical knowledge has been developed and accumulated by the local people, especially knowledge about WEP species. Since WEP species have not been domesticated and grown on a large scale, they must be obtained from the natural environment in order to be used as food $[13,14]$.

In some parts of Yunnan, there has been a rapid replacement of complex agroecosystems by intensive monocultures of commercial crops, such as bananas, which is often accompanied by the use of inorganic herbicides and pesticides [15]. Rising awareness and concern about the possible health effects of pesticides and herbicides on human health has dramatically increased demand for organic foods in China [15]. This interest extends to WEP species as they are wild harvested. For example, many WEP species have made their way into ethnic minority recipes found on highend restaurant menus. Consequently, some WEP species have tremendous market potential and are particularly popular in tourist areas because of their perceived advantages of being pesticide-free, naturally grown, high in nutrients, and fresh in taste $[13,16]$. According to a Web of Science search of bibliometric and mapping knowledge domains, WEP species have always been an essential hotspot

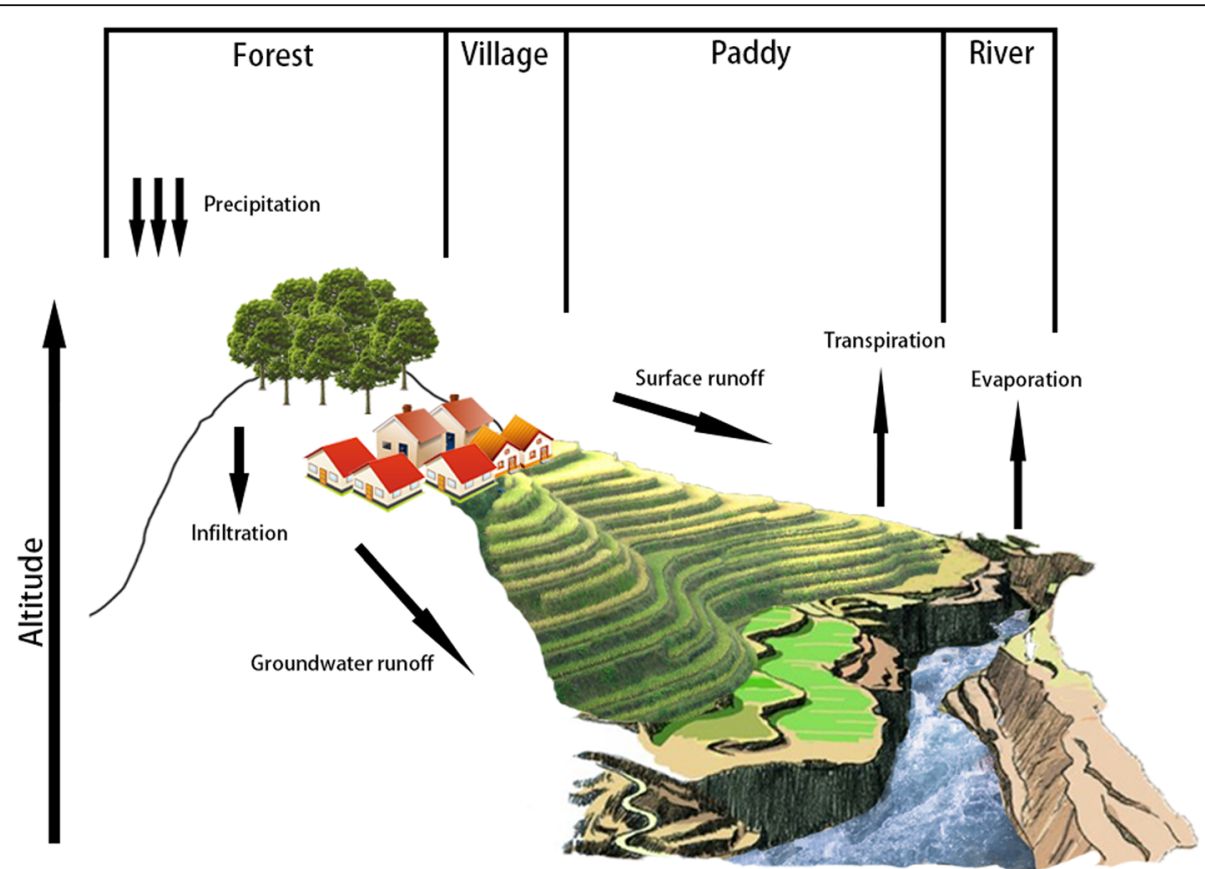

Fig. 1 The construction of the Hani terraced rice paddy field agroecosystem 
in ethnobotanical research [17]. WEP species in the Hani terraced rice paddy agroecosystem are often used to supplement daily food resources or to help to overcome seasonal food shortages [18]. Additionally, some WEP species possess medicinal properties that may help protect indigenous people against diseases $[18,19]$.

Although the Hani people have lived in the Honghe Hani terraced rice paddy system for centuries, their traditional knowledge and associated biodiversity are rapidly being lost due to socio-economic changes and access to modern technologies [4, 11]. Consequently, decreasing traditional knowledge will likely lead to a decrease in biodiversity, especially the diversity of WEP species [20]. Therefore, saving local traditional knowledge and protecting biodiversity are urgent [21]. To our knowledge, no previous studies have documented the WEP species in Hani terraced rice paddy agroecosystems. Thus, this investigation on the WEP species in Hani was conducted. This study recorded traditional knowledge of WEP species, which may protect it from disappearing in a rapid-developing era. The related research results may also provide scientific guidance for WEP species consumption, information of economic benefit to local communities for future sustainable development, and application of WEP species.

\section{Methods}

\section{Study area}

Before the field survey, a literature review was conducted to obtain information about the region of Hani terraced rice paddy fields, including climates, topography, vegetation types, and culture [22]. During 2014-2019, ethnobotanical studies were carried out in four counties (Yuanyang, Lüchun, Honghe, and Jinping), which cover more than 47, 000 ha, including most of the area containing Hani terraced rice paddy fields (Fig. 2) [23]. All study sites and their visit times are recorded in detail in Table 1. In consideration of local landscape diversity, this investigation was conducted in almost every landscape of this agroecosystem, including farming areas, forests, villages, home gardens, and water source areas [23]. Additionally, local markets in different villages and counties were surveyed repeatedly, as the

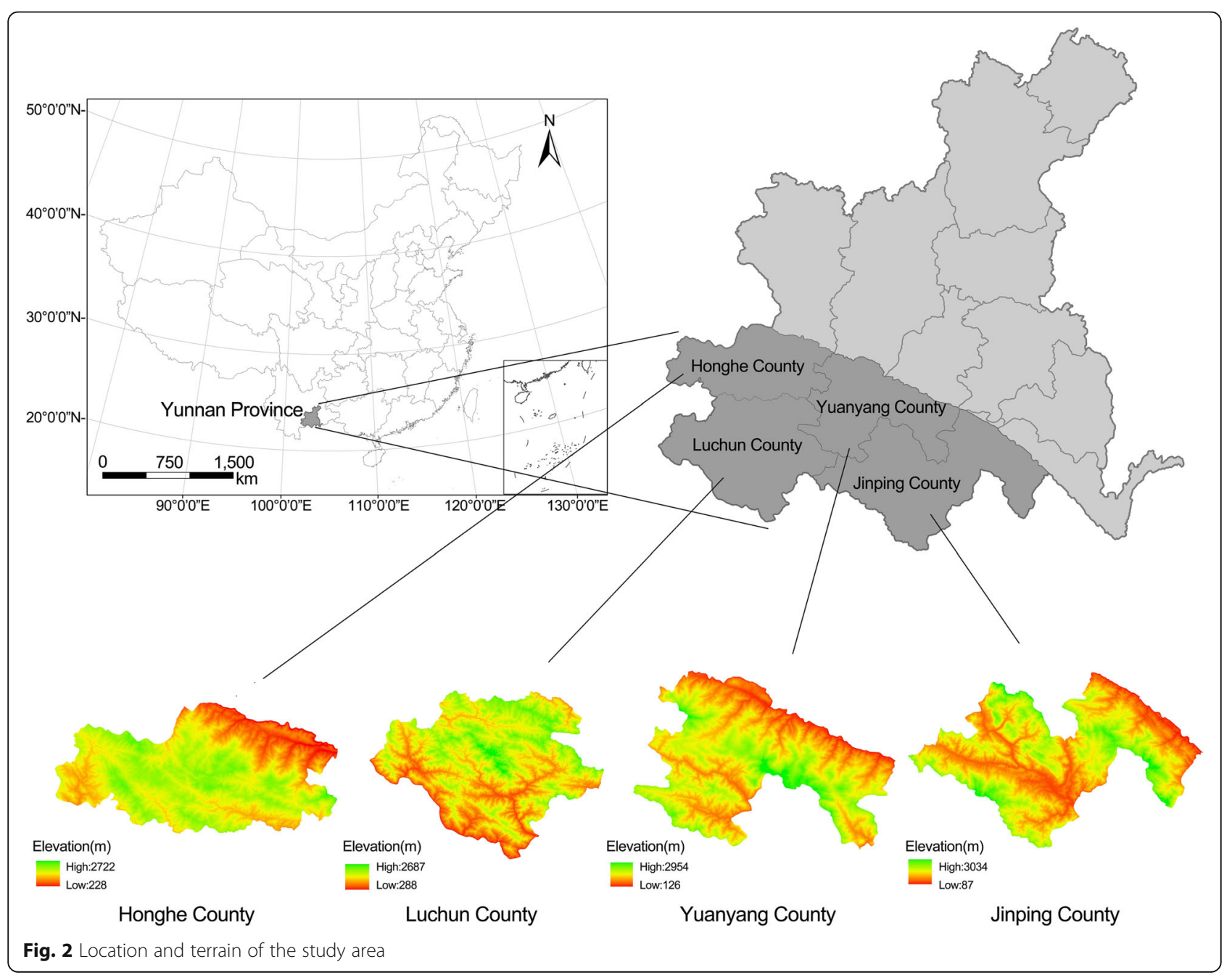


Table 1 Study sites in Honghe Hani terraced rice paddy system

\begin{tabular}{lll}
\hline County name & Village and township & Times visited \\
\hline Honghe County & Lüshuge Village, Jiayin Township & 2 \\
Honghe County & Lonajia Village, Jiayin Township & 1 \\
Honghe County & Baohua Township & 2 \\
Jinping County & Xiongjia Village, Adebo Township & 2 \\
Jinping County & Shuiyan Village, Ma'andi Township & 2 \\
Lüchun County & Lüchun County & 2 \\
Lüchun County & Lagu Village, Sanmeng Township & 1 \\
Yuanyang County & Xiaoxinjie Township & 1 \\
Yuanyang County & Niujiaozhai Township & 1 \\
Yuanyang County & Qingkou Village, Xinjie Township & 1 \\
- & The junction of Lüchun County and Yuanyang County & 1 \\
\hline
\end{tabular}

markets often reflect the wide variety of local knowledge in daily life [24].

\section{Data collection, voucher specimen collection, and data analysis}

A variety of different ethnobotanical and social science methods were used to collect data about the WEP 7 species in this region. These methods included participatory rural appraisal (PRA), direct observation, semi-structured interviews, key informant interviews, and focal group discussions (Fig. 3) [4, 25, 26]. In total, 186 native Hani people, including 160 people older than 50 years of age, were interviewed. Seventy of them were male, and 116 were female. They were mostly local farmers, and many of them collected WEPs to sell in local markets. The primary content of the interview consisted of " $5 \mathrm{~W}+\mathrm{H}$ " questions (i.e., questions concerning what, when, where, who/whom, why, and how the subjects utilize WEP) [24, 26, 27]. With the assistance of Hani local experts, voucher specimens were gathered from different habitats around the study sites. Plant species were identified by Dr. Chunlin Long, Dr. Bo Liu, and Ms. Jun Yang. The voucher specimens were deposited at the College of Life and Environmental Sciences at the Minzu University of China in Beijing.

The data collected in the Honghe area was collated into an inventory containing all the WEP species and related information. The use-value (UV) of each WEP was calculated to evaluate the relative importance of each plant based on the number of times cited and the number of informants $[28,29]$. The formula for UV is

$$
\mathrm{UV}=\left(\sum U_{i}\right) / N
$$

$U_{\mathrm{i}}$ is the times cited by each informant for a certain WEP, while $N$ is the total number of informants [29]. The frequency of utilization index (FUI) of WEP species was graded according to the frequency of consumption by local people. FUI can also reflect the degree of closeness between WEP species and the local community [29]. The FUI scores range from 0 to 5 and varied by the consumption frequency (Table 2) [29].

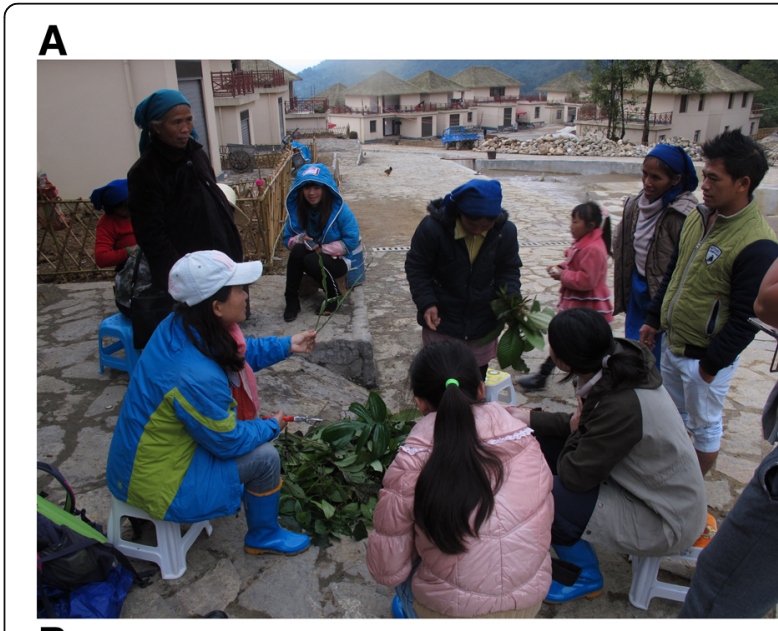

B

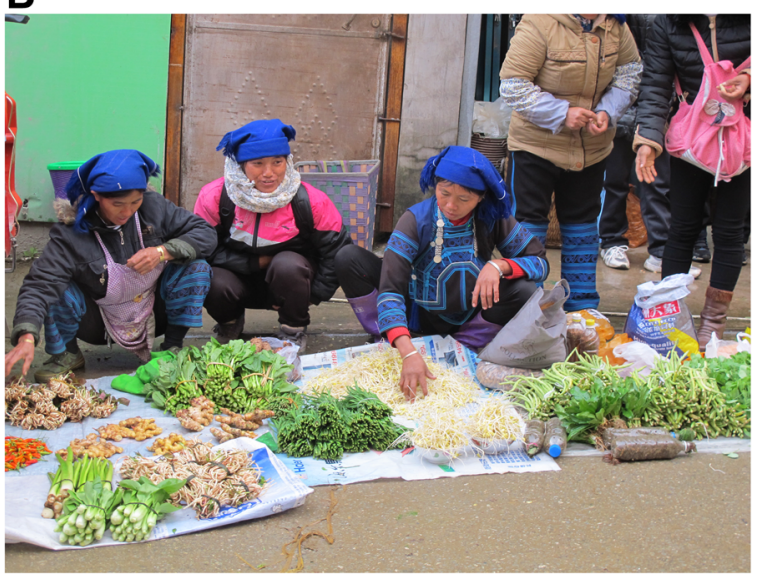

Fig. 3 Focal group discussion (a) and Hani women in a local market (b) 
Table 2 The FUI value and corresponding category

\begin{tabular}{ll}
\hline Consumption frequency & FUI \\
\hline More than once a week & 5 \\
Once a week & 4 \\
Once a month & 3 \\
More than once a year, less than once a month & 2 \\
Once a year & 1 \\
No consumption in last 30 years & 0.5
\end{tabular}

\section{Results and discussion}

Diversity of WEP species in Honghe terraced rice paddy fields

Based on our investigations, 224 WEP species belonging to 170 genera and 90 families, along with related information such as scientific names, family names, life forms, vernacular names, edible parts, and processing methods, were documented (Table 3 ). According to the recorded WEP species, more than half of the species are woody plants $(50.9 \%)$, including trees $(30.4 \%)$ and shrubs (20.5\%). There were 80 species of herbaceous plants (35.3\%), 21 species of lianas (9.4\%), and 10 species of bamboos (4.5\%) (Table 4 ).

All WEP species were also classified by their edible parts (Table 5). The recorded edible parts of WEP species included the whole plant, root, stem and leaf, flower, fruit, seed and shoot, and other parts like bark and tuber. For several WEP species, like Bauhinia acuminata var. candida and Senna tora, multiple parts can be consumed. These results embody the diversity of edible parts of WEP species in Honghe terraced rice paddy fields and indicate that local people have become well adapted to the local environment for centuries. The various uses and preparation methods are recorded in Table 6.

The plant stems and leaves are also collected widely (Table 6), and these are mostly consumed as a potherb, which is generally referred to as "wild vegetables" locally. The Hani people usually consume potherbs by stir-frying or by boiling them in a soup. The blended vegetables in soups are usually mixed with natural spices before eating. There are 16 species with edible flowers (Table 5), including Musa itinerans (bracts only). These edible flowers could be an essential source of nutrition for local people. It has been previously reported that edible flowers are rich in nutrients and micronutrients and that some of their extracts are useful as medicines [30-32]. Potherb is the most consumed group (Table 6) of WEP species in Honghe with 75 species (33.5\%). In China today, wild vegetables, or "ye cai," have become popular food products that are increasingly being served in restaurants due to their flavor and a widespread perception of their superior nutritional values [33]. In the Honghe area, wild vegetables also play a vital role in local livelihood as food and dietary supplements. These wild vegetables are mainly collected in the mountains above the rice paddy fields and forest lands, and the collection time lasts from January to October but mainly occurs in the spring. Some plants, like Houttuynia cordata and Oenanthe javanica, can be collected throughout the year.

According to Table 5, edible fruit is the most popular group (98 species, 43.8\%). These are usually consumed freshly without processing, which is the second most common food preparation method for the Hani WEP species (Table 6). Also, fruits can be consumed in several different ways. For example, Amomum maximum fruits are used locally as a natural spice that can help infirm people regain their appetite; the fruits of Ligustrum sinense are used by the local Hani people to brew a unique alcoholic drink, and Canarium album's fruits can be preserved into pickles.

Ten species of bamboo shoots can be made into different dishes that are high in nutritious fibers. Some WEP species in the Honghe region can also be used as natural flavoring agents (16 species), nuts (12), tea substitutes (11), liquor-brewing ingredients (8), grain substitutes (2), and special tofu (2). Exceptionally, there is only one species, Capparis masaikaii, that is used as a natural sweetener by local communities. The locals usually remove the seed coat and chew the kernel directly. C. masaikaii contains high levels of mabinlin, a sweet protein with 400 times the sweetness of sucrose but with meager calories, and consequently, this plant has a high potential for future application in the food industry [34].

\section{Two special cases of WEP species}

During our investigation, some unique cases of utilizing and processing WEP species were observed. In the Honghe area, Phyllanthus emblica bark is prepared in an unusual way (Fig. 4a). Local Hani people collect the $P$. emblica from mountainous forests, remove the branches, and peel off the outer layer of bark, grating off the bitter-tasting inner bark by using pottery shards. Traditionally, they adjust the bitter taste by mixing rice porridge paste with the tender inner bark. Then, the grated inner bark is mixed with roasted ribs, sliced pork liver, salt, and spices and eaten as a traditional dish. Besides its culinary use, $P$. emblica is also used medicinally for its potential anti-microbial, antioxidant and anti-tumor, hypolipidemic, hypoglycemic, and antihypertensive properties [35].

The traditional preparation of Dioscorea subcalva in cuisine is also distinctive. Local women first peel the thin skin from the D. subcalva tubers (Fig. 4). They then use a special tool to grate the peeled tubers into a container with a hot water-ash solution. When the solution has cooled, all of the grated tubers congeal into a sticky and elastic clump. These clumps can be cut into slices and stir-fried with meat. In another use of D. subcalva, 
Table 3 Inventory of WEP species in Honghe terraced rice paddy system

\begin{tabular}{|c|c|c|c|c|c|c|c|c|c|}
\hline Scientific name & $\begin{array}{l}\text { Vernacular } \\
\text { Name }\end{array}$ & $\begin{array}{l}\text { Life } \\
\text { form }\end{array}$ & Family name & $\begin{array}{l}\text { Parts } \\
\text { used }\end{array}$ & Preparation and uses & Study sites & $\begin{array}{l}\text { Voucher } \\
\text { number }\end{array}$ & FUI & UV \\
\hline \multicolumn{10}{|l|}{ Gymnospermae } \\
\hline Gnetum montanum Markgr. & Wo ni ai xi & Liana & Gnetaceae & Seed & $\begin{array}{l}\text { Cooked thoroughly and } \\
\text { eaten (kernel) }\end{array}$ & $\begin{array}{l}\text { Lüchun } \\
\text { County }\end{array}$ & $\begin{array}{l}201,606- \\
19\end{array}$ & 0.6 & 0.21 \\
\hline Gnetum pendulum C.Y.Cheng & Mang dao & Liana & Gnetaceae & Seed & $\begin{array}{l}\text { Cooked thoroughly and } \\
\text { eaten (kernel) }\end{array}$ & $\begin{array}{l}\text { Lagu Village, } \\
\text { Sanmeng } \\
\text { Township }\end{array}$ & HHD-31 & 0.5 & 0.16 \\
\hline \multicolumn{10}{|l|}{ Angiospermae } \\
\hline $\begin{array}{l}\text { Kadsura coccinea (Lem.) A.C. } \\
\text { Sm. }\end{array}$ & Hei lao hu & Liana & Schisandraceae & Fruit & Ripe fruits are eaten fresh & $\begin{array}{l}\text { Lüshuge } \\
\text { Village, Jiayin } \\
\text { Township }\end{array}$ & $\begin{array}{l}201,610- \\
04\end{array}$ & 2.4 & 0.44 \\
\hline Houttuynia cordata Thunb. & Pa huo & Herb & Saururaceae & Rhizome & $\begin{array}{l}\text { Potherb or flavoring } \\
\text { agent }\end{array}$ & $\begin{array}{l}\text { Lagu Village, } \\
\text { Sanmeng } \\
\text { Township }\end{array}$ & HHD-54 & 4.5 & 0.89 \\
\hline Piper betle $\mathrm{L}$. & $\begin{array}{l}\text { Fa qie wei } \\
\text { niu }\end{array}$ & Liana & Piperaceae & Leaf & Flavoring agent & $\begin{array}{l}\text { Lonajia } \\
\text { Village, Jiayin } \\
\text { Township }\end{array}$ & $\begin{array}{l}201,506- \\
39\end{array}$ & 2.7 & 0.55 \\
\hline $\begin{array}{l}\text { Michelia hedyosperma } \\
\text { Y.W.Law }\end{array}$ & Ma la & Tree & Magnoliaceae & Seed & Flavoring agent & $\begin{array}{l}\text { Lagu Village, } \\
\text { Sanmeng } \\
\text { Township }\end{array}$ & HHD-39 & 2.8 & 0.44 \\
\hline Alphonsea mollis Dunn & & Tree & Annonaceae & Fruit & Ripe fruits are eaten fresh & $\begin{array}{l}\text { Lonajia } \\
\text { Village, Jiayin } \\
\text { Township }\end{array}$ & $\begin{array}{l}201,506- \\
35\end{array}$ & 2.0 & 0.57 \\
\hline $\begin{array}{l}\text { Litsea akoensis var. sasakii } \\
\text { (Kamik.) J.C. Liao }\end{array}$ & & Tree & Lauraceae & Fruit & Flavoring agent & $\begin{array}{l}\text { Lüchun } \\
\text { County }\end{array}$ & $\begin{array}{l}201,606- \\
26\end{array}$ & 2.4 & 0.54 \\
\hline Litsea cubeba (Lour.) Pers. & $\begin{array}{l}\text { Mo ye la } \\
\text { pi }\end{array}$ & Shrub & Lauraceae & Fruit & Flavoring agent & $\begin{array}{l}\text { Shuiyan } \\
\text { Village, } \\
\text { Ma'andi } \\
\text { Township }\end{array}$ & $\begin{array}{l}201,511- \\
12\end{array}$ & 2.0 & 0.54 \\
\hline Litsea pungens Hemsl. & Si bi a si & Tree & Lauraceae & Fruit & Flavoring agent & $\begin{array}{l}\text { Baohua } \\
\text { Township }\end{array}$ & $\begin{array}{l}201,511- \\
43\end{array}$ & 4.9 & 0.92 \\
\hline Acorus gramineus Aiton & Ji xiang & Herb & Acoraceae & $\begin{array}{l}\text { Leaf, } \\
\text { rhizome }\end{array}$ & Flavoring agent & $\begin{array}{l}\text { Shuiyan } \\
\text { Village, } \\
\text { Ma'andi } \\
\text { Township }\end{array}$ & $\begin{array}{l}201,511- \\
13\end{array}$ & 2.8 & 0.46 \\
\hline $\begin{array}{l}\text { Amorphophallus konjac } \\
\text { K.Koch }\end{array}$ & Jia mo & Herb & Araceae & $\begin{array}{l}\text { Tender } \\
\text { leaf, } \\
\text { tuber }\end{array}$ & Making "tofu" & $\begin{array}{l}\text { Niujiaozhai } \\
\text { Township }\end{array}$ & $\begin{array}{l}201,606- \\
09\end{array}$ & 2.9 & 0.43 \\
\hline $\begin{array}{l}\text { Colocasia gigantea (Blume) } \\
\text { Hook. f. }\end{array}$ & Bo ju & Herb & Araceae & Petiole & $\begin{array}{l}\text { Potherb (cooked } \\
\text { thoroughly) }\end{array}$ & $\begin{array}{l}\text { Xiaoxinjie } \\
\text { Township }\end{array}$ & $\begin{array}{l}201,506- \\
06\end{array}$ & 4.0 & 0.80 \\
\hline Sagittaria trifolia L. & Wo qi & Herb & Alismataceae & $\begin{array}{l}\text { Tender } \\
\text { leaf, } \\
\text { rhizome }\end{array}$ & $\begin{array}{l}\text { Potherb (stewed or stir- } \\
\text { fried) }\end{array}$ & $\begin{array}{l}\text { Lüshuge } \\
\text { Village, Jiayin } \\
\text { Township }\end{array}$ & $\begin{array}{l}201,610- \\
24\end{array}$ & 2.4 & 0.46 \\
\hline Dioscorea cirrhosa Lour. & $\begin{array}{l}\text { Ai la ma a } \\
\text { si }\end{array}$ & Liana & Dioscoreaceae & Tuber & $\begin{array}{l}\text { Cereal substitute in } \\
\text { famine time }\end{array}$ & $\begin{array}{l}\text { Xiaoxinjie } \\
\text { Township }\end{array}$ & $\begin{array}{l}201,506- \\
05\end{array}$ & 2.4 & 0.43 \\
\hline $\begin{array}{l}\text { Dioscorea subcalva Prain et } \\
\text { Burkill }\end{array}$ & $\begin{array}{l}\text { Mo mo } \\
\text { mang }\end{array}$ & Liana & Dioscoreaceae & Tuber & $\begin{array}{l}\text { Making "tofu" (similar to } \\
\text { konjac tofu) }\end{array}$ & $\begin{array}{l}\text { Lagu Village, } \\
\text { Sanmeng } \\
\text { Township }\end{array}$ & HHD-45 & 2.6 & 0.46 \\
\hline $\begin{array}{l}\text { Heterosmilax yunnanensis } \\
\text { Gagnep. }\end{array}$ & $\begin{array}{l}\text { Guo ge } \\
\text { niao, a } \\
\text { guo guo } \\
\text { ne }\end{array}$ & Shrub & Smilacaceae & $\begin{array}{l}\text { Tender } \\
\text { leaf }\end{array}$ & $\begin{array}{l}\text { Potherb (blanched in } \\
\text { boiled water, then } \\
\text { soaked in cold water for } \\
\text { days. Usually stir-fried or } \\
\text { made into soup) }\end{array}$ & $\begin{array}{l}\text { The junction } \\
\text { of Lüchun } \\
\text { County and } \\
\text { Yuanyang } \\
\text { County }\end{array}$ & $\begin{array}{l}201,506- \\
08\end{array}$ & 2.7 & 0.47 \\
\hline Caryota urens L. & $\begin{array}{l}\text { Ha da a } \\
\text { bo }\end{array}$ & Tree & Arecaceae & Flower & $\begin{array}{l}\text { Snack (inflorescence sap } \\
\text { is sweet) }\end{array}$ & $\begin{array}{l}\text { Lüshuge } \\
\text { Village, Jiayin } \\
\text { Township }\end{array}$ & $\begin{array}{l}201,610- \\
13\end{array}$ & 0.9 & 0.20 \\
\hline Commelina benghalensis & A wei ya & Herb & Commelinaceae & Tender & Potherb (boiled for 5-10 & Lüchun & LB-27 & 2.0 & 0.49 \\
\hline
\end{tabular}


Table 3 Inventory of WEP species in Honghe terraced rice paddy system (Continued)

\begin{tabular}{|c|c|c|c|c|c|c|c|c|c|}
\hline Scientific name & $\begin{array}{l}\text { Vernacular } \\
\text { Name }\end{array}$ & $\begin{array}{l}\text { Life } \\
\text { form }\end{array}$ & Family name & $\begin{array}{l}\text { Parts } \\
\text { used }\end{array}$ & Preparation and uses & Study sites & $\begin{array}{l}\text { Voucher } \\
\text { number }\end{array}$ & FUI & UV \\
\hline Forssk. & mo & & & $\begin{array}{l}\text { Leaf, } \\
\text { tender } \\
\text { stem }\end{array}$ & $\begin{array}{l}\text { min, then soaked in } \\
\text { water to debitterize) }\end{array}$ & County & & & \\
\hline Commelina diffusa Burm.f. & $\begin{array}{l}\text { Nuo niu } \\
\text { pao }\end{array}$ & Herb & Commelinaceae & $\begin{array}{l}\text { Whole } \\
\text { plant }\end{array}$ & $\begin{array}{l}\text { Potherb (usually stewed } \\
\text { with pork) }\end{array}$ & $\begin{array}{l}\text { Lüshuge } \\
\text { Village, Jiayin } \\
\text { Township }\end{array}$ & $\begin{array}{l}201,610- \\
15\end{array}$ & 2.9 & 0.42 \\
\hline Streptolirion volubile Edgew. & $\begin{array}{l}\text { Mo dui dui } \\
\text { han }\end{array}$ & Herb & Commelinaceae & $\begin{array}{l}\text { Tender } \\
\text { stem, } \\
\text { leaf }\end{array}$ & $\begin{array}{l}\text { Potherb (made into } \\
\text { soup) }\end{array}$ & $\begin{array}{l}\text { Xiaoxinjie } \\
\text { Township }\end{array}$ & LB-16 & 2.2 & 0.56 \\
\hline $\begin{array}{l}\text { Monochoria vaginalis (Burm.f.) } \\
\text { C.Presl }\end{array}$ & $\begin{array}{l}\text { Mi zuo wa, } \\
\text { a bei bei } \\
\text { za, e za, e } \\
\text { bi ra }\end{array}$ & Herb & Pontederiaceae & $\begin{array}{l}\text { Stem } \\
\text { and leaf }\end{array}$ & Potherb & $\begin{array}{l}\text { Lagu Village, } \\
\text { Sanmeng } \\
\text { Township }\end{array}$ & HHD-48 & 2.0 & 0.47 \\
\hline Musa acuminata Colla & $\begin{array}{l}\text { Ruo a pao } \\
\text { ruo a wo }\end{array}$ & Herb & Musaceae & $\begin{array}{l}\text { Fruit, } \\
\text { flower, } \\
\text { pith part }\end{array}$ & $\begin{array}{l}\text { Fruit: eaten fresh; flower } \\
\text { and pith part: cooked as } \\
\text { potherb }\end{array}$ & $\begin{array}{l}\text { Baohua } \\
\text { Township }\end{array}$ & $\begin{array}{l}201,511- \\
44\end{array}$ & 3.8 & 0.70 \\
\hline Musa itinerans Cheesman & & Herb & Musaceae & $\begin{array}{l}\text { Flower, } \\
\text { young } \\
\text { bract }\end{array}$ & Potherb & $\begin{array}{l}\text { Xiaoxinjie } \\
\text { Township }\end{array}$ & LB-14 & 2.6 & 0.41 \\
\hline Amomum maximum Roxb. & $\begin{array}{l}\text { Sa jia } \\
\text { hong bi }\end{array}$ & Herb & Zingiberaceae & Fruit & Flavoring agent & $\begin{array}{l}\text { Lüshuge } \\
\text { Village, Jiayin } \\
\text { Township }\end{array}$ & $\begin{array}{l}201,610- \\
12\end{array}$ & 2.7 & 0.52 \\
\hline $\begin{array}{l}\text { Hedychium coronarium } \\
\text { J.Koenig }\end{array}$ & A ci a ye & Herb & Zingiberaceae & $\begin{array}{l}\text { Flower, } \\
\text { shoot }\end{array}$ & $\begin{array}{l}\text { Potherb (usually stewed } \\
\text { or stir fried) }\end{array}$ & $\begin{array}{l}\text { Lonajia } \\
\text { Village, Jiayin } \\
\text { Township }\end{array}$ & $\begin{array}{l}201,506- \\
53\end{array}$ & 2.3 & 0.45 \\
\hline Zingiber striolatum Diels & & Herb & Zingiberaceae & Flower & Potherb & $\begin{array}{l}\text { Lüshuge } \\
\text { Village, Jiayin } \\
\text { Township }\end{array}$ & $\begin{array}{l}201,610- \\
22\end{array}$ & 3.0 & 0.73 \\
\hline $\begin{array}{l}\text { Acidosasa hirtiflora Z.P.Wang } \\
\text { and G.H.Ye }\end{array}$ & & Bamboo & Poaceae & Shoot & Bamboo shoots & $\begin{array}{l}\text { Shuiyan } \\
\text { Village, } \\
\text { Ma'andi } \\
\text { Township }\end{array}$ & $\begin{array}{l}201,511- \\
20\end{array}$ & 0.1 & 0.12 \\
\hline $\begin{array}{l}\text { Chimonobambusa } \\
\text { yunnanensis Hsueh et W.P. } \\
\text { Zhang }\end{array}$ & & Bamboo & Poaceae & Shoot & Bamboo shoots & $\begin{array}{l}\text { Shuiyan } \\
\text { Village, } \\
\text { Ma'andi } \\
\text { Township }\end{array}$ & $\begin{array}{l}201,511- \\
22\end{array}$ & 1.0 & 0.18 \\
\hline $\begin{array}{l}\text { Chimonocalamus } \\
\text { longiligulatus Hsueh and } \\
\text { T.P.Yi }\end{array}$ & Ha bo & Bamboo & Poaceae & Shoot & Bamboo shoots & $\begin{array}{l}\text { Xiaoxinjie } \\
\text { Township }\end{array}$ & LB-07 & 0.5 & 0.18 \\
\hline $\begin{array}{l}\text { Dendrocalamus } \\
\text { membranaceus Munro }\end{array}$ & A ha a bi & Bamboo & Poaceae & Shoot & Bamboo shoots & $\begin{array}{l}\text { Xiongjia } \\
\text { Village, } \\
\text { Adebo } \\
\text { Township }\end{array}$ & $\begin{array}{l}\text { HHD- } \\
015\end{array}$ & 0.6 & 0.17 \\
\hline $\begin{array}{l}\text { Dendrocalamus peculiaris } \\
\text { Hsueh and D.Z.Li }\end{array}$ & & Bamboo & Poaceae & Shoot & Bamboo shoots & $\begin{array}{l}\text { Shuiyan } \\
\text { Village, } \\
\text { Ma'andi } \\
\text { Township }\end{array}$ & $\begin{array}{l}201,511- \\
23\end{array}$ & 0.8 & 0.25 \\
\hline $\begin{array}{l}\text { Indosasa singulispicula } \\
\text { T.H.Wen }\end{array}$ & & Bamboo & Poaceae & Shoot & Bamboo shoots & $\begin{array}{l}\text { Lonajia } \\
\text { Village, Jiayin } \\
\text { Township }\end{array}$ & $\begin{array}{l}201,506- \\
43\end{array}$ & 0.1 & 0.15 \\
\hline $\begin{array}{l}\text { Indosasa sinica C.D.Chu and } \\
\text { C.S.Chao }\end{array}$ & A xiu a bo & Bamboo & Poaceae & Shoot & Bamboo shoots & $\begin{array}{l}\text { Shuiyan } \\
\text { Village, } \\
\text { Ma'andi } \\
\text { Township }\end{array}$ & $\begin{array}{l}201,511- \\
17\end{array}$ & 0.6 & 0.06 \\
\hline Melocalamus arrectus T.P.Yi & A ha a bo & Bamboo & Poaceae & Shoot & Bamboo shoots & $\begin{array}{l}\text { Shuiyan } \\
\text { Village, } \\
\text { Ma'andi } \\
\text { Township }\end{array}$ & $\begin{array}{l}201,511- \\
18\end{array}$ & 0.6 & 0.09 \\
\hline
\end{tabular}


Table $\mathbf{3}$ Inventory of WEP species in Honghe terraced rice paddy system (Continued)

\begin{tabular}{|c|c|c|c|c|c|c|c|c|c|}
\hline Scientific name & $\begin{array}{l}\text { Vernacular } \\
\text { Name }\end{array}$ & $\begin{array}{l}\text { Life } \\
\text { form }\end{array}$ & Family name & $\begin{array}{l}\text { Parts } \\
\text { used }\end{array}$ & Preparation and uses & Study sites & $\begin{array}{l}\text { Voucher } \\
\text { number }\end{array}$ & FUI & UV \\
\hline $\begin{array}{l}\text { Phyllostachys nigra var. } \\
\text { henonis (Mitford) Rendle }\end{array}$ & $\begin{array}{l}\text { A mao a } \\
\text { bo }\end{array}$ & Bamboo & Poaceae & Shoot & Bamboo shoots & $\begin{array}{l}\text { Shuiyan } \\
\text { Village, } \\
\text { Ma'andi } \\
\text { Township }\end{array}$ & $\begin{array}{l}201,511- \\
21\end{array}$ & 0.9 & 0.04 \\
\hline $\begin{array}{l}\text { Schizostachyum funghomii } \\
\text { McClure }\end{array}$ & A che & Bamboo & Poaceae & Shoot & Bamboo shoots & $\begin{array}{l}\text { Shuiyan } \\
\text { Village, } \\
\text { Ma'andi } \\
\text { Township }\end{array}$ & $\begin{array}{l}201,511- \\
19\end{array}$ & 0.5 & 0.25 \\
\hline $\begin{array}{l}\text { Akebia trifoliata (Thunb.) } \\
\text { Koidz. }\end{array}$ & & Liana & Lardizabalaceae & Fruit & Ripe fruits are eaten fresh & $\begin{array}{l}\text { Lüshuge } \\
\text { Village, Jiayin } \\
\text { Township }\end{array}$ & $\begin{array}{l}201,610- \\
05\end{array}$ & 1.9 & 0.54 \\
\hline Parabaena sagittata Miers & $\begin{array}{l}\text { Hua na } \\
\text { wei niu }\end{array}$ & Liana & Menispermaceae & Leaf & Potherb & $\begin{array}{l}\text { Shuiyan } \\
\text { Village, } \\
\text { Ma'andi } \\
\text { Township }\end{array}$ & $\begin{array}{l}201,511- \\
28\end{array}$ & 2.5 & 0.55 \\
\hline $\begin{array}{l}\text { Mahonia bealei (Fortune) } \\
\text { Pynaert }\end{array}$ & $\begin{array}{l}\text { Shi shi, } \\
\text { sou shou }\end{array}$ & Shrub & Berberidaceae & $\begin{array}{l}\text { Fruit, } \\
\text { stem }\end{array}$ & $\begin{array}{l}\text { Stem: liquor brewing; } \\
\text { fruit: eaten fresh }\end{array}$ & $\begin{array}{l}\text { Lonajia } \\
\text { Village, Jiayin } \\
\text { Township }\end{array}$ & $\begin{array}{l}201,506- \\
45\end{array}$ & 2.6 & 0.52 \\
\hline Helicia nilagirica Bedd. & $\begin{array}{l}\text { Kong bai a } \\
\text { bo }\end{array}$ & Tree & Proteaceae & Seed & $\begin{array}{l}\text { Cooked seeds are used } \\
\text { as grain substitute }\end{array}$ & $\begin{array}{l}\text { Shuiyan } \\
\text { Village, } \\
\text { Ma'andi } \\
\text { Township }\end{array}$ & $\begin{array}{l}201,511- \\
01\end{array}$ & 2.7 & 0.58 \\
\hline Dillenia indica L. & Xi shi a di & Tree & Dilleniaceae & Fruit & Ripe fruits are eaten fresh & $\begin{array}{l}\text { Qingkou } \\
\text { Village, Xinjie } \\
\text { Township }\end{array}$ & $\begin{array}{l}201,506- \\
13\end{array}$ & 2.8 & 0.41 \\
\hline Acacia pennata (L.) Willd. & $\begin{array}{l}\text { Tuo bo ji } \\
\text { niu }\end{array}$ & Liana & Fabaceae & $\begin{array}{l}\text { Tender } \\
\text { leaf }\end{array}$ & Potherb & $\begin{array}{l}\text { Baohua } \\
\text { Township }\end{array}$ & HHD-25 & 3.4 & 0.65 \\
\hline $\begin{array}{l}\text { Bauhinia acuminata L. var. } \\
\text { candida (Roxb.) Voigt }\end{array}$ & $\begin{array}{l}\text { Du bie a } \\
\text { lo }\end{array}$ & Shrub & Fabaceae & $\begin{array}{l}\text { Flower, } \\
\text { young } \\
\text { pod, } \\
\text { seed, } \\
\text { tender } \\
\text { leaf }\end{array}$ & $\begin{array}{l}\text { Potherb, seeds: cooked } \\
\text { throughly and eaten } \\
\text { (kernel) }\end{array}$ & $\begin{array}{l}\text { Xiaoxinjie } \\
\text { Township }\end{array}$ & LB-17 & 2.9 & 0.40 \\
\hline $\begin{array}{l}\text { Chamaecrista mimosoides (L.) } \\
\text { Greene }\end{array}$ & & Herb & Fabaceae & $\begin{array}{l}\text { Tender } \\
\text { leaf }\end{array}$ & Tea substitute & $\begin{array}{l}\text { Qingkou } \\
\text { Village, Xinjie } \\
\text { Township }\end{array}$ & $\begin{array}{l}201,506- \\
19\end{array}$ & 0.5 & 0.14 \\
\hline $\begin{array}{l}\text { Chamaecrista nictitans (L.) } \\
\text { Moench subsp. patellaris (DC. } \\
\text { ex Collad.) H. S. Irwin et } \\
\text { Barneby var. glabrata (Vogel) } \\
\text { H. S. Irwin et Barneby }\end{array}$ & & Herb & Fabaceae & $\begin{array}{l}\text { Tender } \\
\text { leaf, } \\
\text { tender } \\
\text { stem }\end{array}$ & Tea substitute & $\begin{array}{l}\text { Lüchun } \\
\text { County }\end{array}$ & LB-18 & 1.2 & 0.30 \\
\hline Gleditsia sinensis Lam. & $\begin{array}{l}\text { A si ni ma } \\
\text { a hong }\end{array}$ & Tree & Fabaceae & $\begin{array}{l}\text { Tender } \\
\text { leaf }\end{array}$ & Potherb & $\begin{array}{l}\text { Lüshuge } \\
\text { Village, Jiayin } \\
\text { Township }\end{array}$ & $\begin{array}{l}201,610- \\
20\end{array}$ & 2.7 & 0.48 \\
\hline Parochetus communis D.Don & $\begin{array}{l}\text { A wo la } \\
\text { gian }\end{array}$ & Herb & Fabaceae & Flower & Potherb (stir-fried) & $\begin{array}{l}\text { Lüchun } \\
\text { County }\end{array}$ & $\begin{array}{l}201,606- \\
31\end{array}$ & 2.5 & 0.57 \\
\hline Senna tora (L.) Roxb. & & Herb & Fabaceae & $\begin{array}{l}\text { Flower, } \\
\text { leaf, } \\
\text { young } \\
\text { fruit, } \\
\text { seed }\end{array}$ & $\begin{array}{l}\text { Potherb, seed: substitute } \\
\text { of coffee }\end{array}$ & $\begin{array}{l}\text { Lüchun } \\
\text { County }\end{array}$ & LB-28 & 2.6 & 0.48 \\
\hline $\begin{array}{l}\text { Tadehagi triquetrum (L.) } \\
\text { H.Ohashi }\end{array}$ & $\begin{array}{l}\text { Qian ka a } \\
\text { bo }\end{array}$ & Shrub & Fabaceae & $\begin{array}{l}\text { Tender } \\
\text { leaf, } \\
\text { tender } \\
\text { stem }\end{array}$ & Tea substitute & $\begin{array}{l}\text { Lagu Village, } \\
\text { Sanmeng } \\
\text { Township }\end{array}$ & HHD-43 & 0.7 & 0.23 \\
\hline Tamarindus indica L. & Bi qian a si & Tree & Fabaceae & $\begin{array}{l}\text { Fruit, } \\
\text { tender } \\
\text { leaf }\end{array}$ & $\begin{array}{l}\text { Fruit: eaten fresh or } \\
\text { made into compote; } \\
\text { tender leaf: potherb } \\
\text { (blanched before }\end{array}$ & $\begin{array}{l}\text { Niujiaozhai } \\
\text { Township }\end{array}$ & $\begin{array}{l}201,606- \\
08\end{array}$ & 2.5 & 0.56 \\
\hline
\end{tabular}


Table 3 Inventory of WEP species in Honghe terraced rice paddy system (Continued)

\begin{tabular}{|c|c|c|c|c|c|c|c|c|c|}
\hline Scientific name & $\begin{array}{l}\text { Vernacular } \\
\text { Name }\end{array}$ & $\begin{array}{l}\text { Life } \\
\text { form }\end{array}$ & Family name & $\begin{array}{l}\text { Parts } \\
\text { used }\end{array}$ & Preparation and uses & Study sites & $\begin{array}{l}\text { Voucher } \\
\text { number }\end{array}$ & FUI & UV \\
\hline & & & & & cooking) & & & & \\
\hline $\begin{array}{l}\text { Fagopyrum dibotrys (D.Don) } \\
\text { H.Hara }\end{array}$ & A za ca sa & Herb & Polygonaceae & Root & $\begin{array}{l}\text { Potherb (usually made } \\
\text { into soup) }\end{array}$ & $\begin{array}{l}\text { Xiongjia } \\
\text { Village, } \\
\text { Adebo } \\
\text { Township }\end{array}$ & $\begin{array}{l}201,506- \\
04\end{array}$ & 2.2 & 0.57 \\
\hline Polygala fallax Hemsl. & $\begin{array}{l}\text { Ha pa ha } \\
\text { ma }\end{array}$ & Shrub & Polygalaceae & $\begin{array}{l}\text { Flower, } \\
\text { tender } \\
\text { leaf }\end{array}$ & $\begin{array}{l}\text { Potherb (usually made } \\
\text { into soup) }\end{array}$ & $\begin{array}{l}\text { Lonajia } \\
\text { Village, Jiayin } \\
\text { Township }\end{array}$ & $\begin{array}{l}201,506- \\
52\end{array}$ & 2.3 & 0.47 \\
\hline $\begin{array}{l}\text { Polygonum cupitatum Buch.- } \\
\text { Ham. ex D.Don }\end{array}$ & A za za ni & Herb & Polygonaceae & $\begin{array}{l}\text { Tender } \\
\text { leaf }\end{array}$ & $\begin{array}{l}\text { Potherb (usually made } \\
\text { into soup) }\end{array}$ & $\begin{array}{l}\text { Xiongjia } \\
\text { Village, } \\
\text { Adebo } \\
\text { Township }\end{array}$ & $\begin{array}{l}201,506- \\
03\end{array}$ & 2.0 & 0.46 \\
\hline Polygonum hydropiper $\mathrm{L}$. & $\begin{array}{l}\text { An ji ba } \\
\text { qian }\end{array}$ & Herb & Polygonaceae & $\begin{array}{l}\text { Tender } \\
\text { leaf, } \\
\text { tender } \\
\text { stem }\end{array}$ & Potherb & $\begin{array}{l}\text { Qingkou } \\
\text { Village, Xinjie } \\
\text { Township }\end{array}$ & $\begin{array}{l}201,506- \\
24\end{array}$ & 2.8 & 0.54 \\
\hline Polygonum molle D. Don & $\begin{array}{l}\text { Qian ge a } \\
\text { si }\end{array}$ & Shrub & Polygonaceae & Fruit & Ripe fruits are eaten fresh & $\begin{array}{l}\text { Baohua } \\
\text { Township }\end{array}$ & $\begin{array}{l}201,511- \\
33\end{array}$ & 2.2 & 0.50 \\
\hline Polygonum perfoliatum L. & $\begin{array}{l}\text { A qian la } \\
\text { qian a pa }\end{array}$ & Herb & Polygonaceae & Fruit & Ripe fruits are eaten fresh & $\begin{array}{l}\text { Xiaoxinjie } \\
\text { Township }\end{array}$ & LB-03 & 2.1 & 0.58 \\
\hline Reynoutria japonica Houtt. & $\begin{array}{l}\text { Suan gan } \\
\text { tong }\end{array}$ & Herb & Polygonaceae & $\begin{array}{l}\text { Tender } \\
\text { stem }\end{array}$ & Potherb & $\begin{array}{l}\text { Lonajia } \\
\text { Village, Jiayin } \\
\text { Township }\end{array}$ & $\begin{array}{l}201,506- \\
37\end{array}$ & 2.0 & 0.58 \\
\hline $\begin{array}{l}\text { Xanthophyllum yunnanense } \\
\text { C.Y. Wu }\end{array}$ & & Tree & Polygalaceae & Fruit & Ripe fruits are eaten fresh & $\begin{array}{l}\text { Lüshuge } \\
\text { Village, Jiayin } \\
\text { Township }\end{array}$ & $\begin{array}{l}201,610- \\
07\end{array}$ & 2.7 & 0.42 \\
\hline Crataegus pinnatifida Bunge & Si pu a si & Tree & Rosaceae & Fruit & Ripe fruits are eaten fresh & $\begin{array}{l}\text { Baohua } \\
\text { Township }\end{array}$ & $\begin{array}{l}201,511- \\
37\end{array}$ & 1.8 & 0.38 \\
\hline Fragaria vesca $\mathrm{L}$. & $\begin{array}{l}\text { O luo jia } \\
\text { ba a si }\end{array}$ & Herb & Rosaceae & Fruit & Ripe fruits are eaten fresh & $\begin{array}{l}\text { Lüshuge } \\
\text { Village, Jiayin } \\
\text { Township }\end{array}$ & $\begin{array}{l}201,610- \\
08\end{array}$ & 2.1 & 0.44 \\
\hline Pyrus calleryana Decne. & $\begin{array}{l}\text { Si peng a } \\
\text { si }\end{array}$ & Tree & Rosaceae & $\begin{array}{l}\text { Flower, } \\
\text { fruit }\end{array}$ & $\begin{array}{l}\text { Fruit: eaten fresh; flower: } \\
\text { potherb (soaked in water } \\
\text { to dibitterize, then stir- } \\
\text { fried, made into soup or } \\
\text { salad) }\end{array}$ & $\begin{array}{l}\text { Lonajia } \\
\text { Village, Jiayin } \\
\text { Township }\end{array}$ & $\begin{array}{l}201,506- \\
41\end{array}$ & 2.0 & 0.54 \\
\hline Pyrus xerophila T.T.Yu & $\begin{array}{l}\text { A pei pei } \\
\text { zi zuo }\end{array}$ & Tree & Rosaceae & Fruit & Ripe fruits are eaten fresh & $\begin{array}{l}\text { Lüchun } \\
\text { County }\end{array}$ & $\begin{array}{l}201,606- \\
22\end{array}$ & 2.7 & 0.49 \\
\hline $\begin{array}{l}\text { Rubus ellipticus var. } \\
\text { obcordatus (Franch.) Focke }\end{array}$ & Huo wo & Shrub & Rosaceae & Fruit & Ripe fruits are eaten fresh & $\begin{array}{l}\text { Lüchun } \\
\text { County }\end{array}$ & $\begin{array}{l}201,606- \\
21\end{array}$ & 2.7 & 0.38 \\
\hline $\begin{array}{l}\text { Rubus multibracteatus H. Lév. } \\
\text { and Vaniot }\end{array}$ & & Shrub & Rosaceae & Fruit & Ripe fruits are eaten fresh & $\begin{array}{l}\text { Lagu Village, } \\
\text { Sanmeng } \\
\text { Township }\end{array}$ & HHD-33 & 1.9 & 0.58 \\
\hline Rubus parvifolius L. & $\begin{array}{l}\text { A guo luo } \\
\text { a bei }\end{array}$ & Shrub & Rosaceae & Fruit & Ripe fruits are eaten fresh & $\begin{array}{l}\text { Qingkou } \\
\text { Village, Xinjie } \\
\text { Township }\end{array}$ & $\begin{array}{l}201,506- \\
15\end{array}$ & 2.5 & 0.56 \\
\hline Elaeagnus conferta Roxb. & $\begin{array}{l}\text { Ba pen luo } \\
\text { niu }\end{array}$ & Shrub & Elaeagnaceae & Fruit & Ripe fruits are eaten fresh & $\begin{array}{l}\text { Lonajia } \\
\text { Village, Jiayin } \\
\text { Township }\end{array}$ & $\begin{array}{l}201,506- \\
30\end{array}$ & 2.5 & 0.41 \\
\hline $\begin{array}{l}\text { Artocarpus lacucha Buch.- } \\
\text { Ham. ex D. Don }\end{array}$ & $\begin{array}{l}\text { A niao } \\
\text { niao bei }\end{array}$ & Tree & Moraceae & Fruit & Ripe fruits are eaten fresh & $\begin{array}{l}\text { Lüshuge } \\
\text { Village, Jiayin } \\
\text { Township }\end{array}$ & $\begin{array}{l}201,610- \\
09\end{array}$ & 2.9 & 0.57 \\
\hline $\begin{array}{l}\text { Artocarpus tonkinensis A.Chev. } \\
\text { ex Gagnep. }\end{array}$ & $\begin{array}{l}\text { Ci gan gan } \\
\text { nü }\end{array}$ & Tree & Moraceae & Fruit & Ripe fruits are eaten fresh & $\begin{array}{l}\text { Lonajia } \\
\text { Village, Jiayin } \\
\text { Township }\end{array}$ & $\begin{array}{l}201,506- \\
29\end{array}$ & 2.3 & 0.47 \\
\hline $\begin{array}{l}\text { Broussonetia papyrifera }(\mathrm{L} .) \\
\text { L'Her.ex Vent. }\end{array}$ & Ma san & Tree & Moraceae & $\begin{array}{l}\text { Flower, } \\
\text { tender }\end{array}$ & Potherb & $\begin{array}{l}\text { Niujiaozhai } \\
\text { Township }\end{array}$ & $\begin{array}{l}201,606- \\
16\end{array}$ & 2.0 & 0.44 \\
\hline
\end{tabular}


Table 3 Inventory of WEP species in Honghe terraced rice paddy system (Continued)

\begin{tabular}{|c|c|c|c|c|c|c|c|c|c|}
\hline Scientific name & $\begin{array}{l}\text { Vernacular } \\
\text { Name }\end{array}$ & $\begin{array}{l}\text { Life } \\
\text { form }\end{array}$ & Family name & $\begin{array}{l}\text { Parts } \\
\text { used }\end{array}$ & Preparation and uses & Study sites & $\begin{array}{l}\text { Voucher } \\
\text { number }\end{array}$ & FUI & UV \\
\hline & & & & leaf & & & & & \\
\hline Ficus auriculata Lour. & $\begin{array}{l}\text { Mu gua } \\
\text { cai }\end{array}$ & Tree & Moraceae & Fruit & Ripe fruits are eaten fresh & $\begin{array}{l}\text { Lüchun } \\
\text { County }\end{array}$ & $\begin{array}{l}201,606- \\
23\end{array}$ & 3.4 & 0.68 \\
\hline Ficus hederacea Roxb. & $\begin{array}{l}\text { Jia ni ni } \\
\text { bai }\end{array}$ & Shrub & Moraceae & Fruit & $\begin{array}{l}\text { Ripe fruits are mixed with } \\
\text { salt and eaten fresh }\end{array}$ & $\begin{array}{l}\text { Shuiyan } \\
\text { Village, } \\
\text { Ma'andi } \\
\text { Township }\end{array}$ & $\begin{array}{l}201,511- \\
16\end{array}$ & 1.9 & 0.55 \\
\hline Ficus henryi Warb. ex Diels & $\begin{array}{l}\text { A niao } \\
\text { niao xiu }\end{array}$ & Tree & Moraceae & Fruit & $\begin{array}{l}\text { Fruits eaten fresh or } \\
\text { liquor brewing }\end{array}$ & $\begin{array}{l}\text { Baohua } \\
\text { Township }\end{array}$ & $\begin{array}{l}201,511- \\
40\end{array}$ & 1.7 & 0.41 \\
\hline Ficus hirta Vahl & Ji zi o si & Shrub & Moraceae & Fruit & Ripe fruits are eaten fresh & $\begin{array}{l}\text { Lagu Village, } \\
\text { Sanmeng } \\
\text { Township }\end{array}$ & HHD-34 & 2.0 & 0.38 \\
\hline Ficus irisana Elmer & Qi pu & Tree & Moraceae & Fruit & Ripe fruits are eaten fresh & $\begin{array}{l}\text { Xiongjia } \\
\text { Village, } \\
\text { Adebo } \\
\text { Township }\end{array}$ & $\begin{array}{l}\text { HHD- } \\
012\end{array}$ & 1.8 & 0.39 \\
\hline Ficus oligodon Miq. & Xi bo ai xi & Tree & Moraceae & Fruit & Ripe fruits are eaten fresh & $\begin{array}{l}\text { Lüshuge } \\
\text { Village, Jiayin } \\
\text { Township }\end{array}$ & $\begin{array}{l}201,610- \\
02\end{array}$ & 2.1 & 0.46 \\
\hline Ficus pandurata Hance & & Shrub & Moraceae & $\begin{array}{l}\text { Fruit, } \\
\text { seed }\end{array}$ & $\begin{array}{l}\text { Fruit: eaten fresh; seed: } \\
\text { roasted and eaten } \\
\text { (kernel) }\end{array}$ & $\begin{array}{l}\text { Lüchun } \\
\text { County }\end{array}$ & $\begin{array}{l}201,606- \\
28\end{array}$ & 2.1 & 0.48 \\
\hline Ficus racemosa $\mathrm{L}$. & $\begin{array}{l}\text { A niao } \\
\text { niao na }\end{array}$ & Tree & Moraceae & $\begin{array}{l}\text { Fruit, } \\
\text { seed }\end{array}$ & $\begin{array}{l}\text { Fruit: eaten fresh; seed: } \\
\text { roasted and eaten } \\
\text { (kernel) }\end{array}$ & $\begin{array}{l}\text { Lüchun } \\
\text { County }\end{array}$ & $\begin{array}{l}201,606- \\
27\end{array}$ & 2.1 & 0.51 \\
\hline $\begin{array}{l}\text { Ficus semicordata Buch.-Ham. } \\
\text { Ex Sm. }\end{array}$ & $\begin{array}{l}\text { Hu gan da } \\
\text { pa }\end{array}$ & Tree & Moraceae & Fruit & Ripe fruits are eaten fresh & $\begin{array}{l}\text { Lagu Village, } \\
\text { Sanmeng } \\
\text { Township }\end{array}$ & HHD-35 & 2.4 & 0.44 \\
\hline Ficus tikona Bureau & $\begin{array}{l}\text { Wei chao } \\
\text { lao e }\end{array}$ & Liana & Moraceae & Fruit & Ripe fruits are eaten fresh & $\begin{array}{l}\text { Xiaoxinjie } \\
\text { Township }\end{array}$ & LB-01 & 2.0 & 0.48 \\
\hline $\begin{array}{l}\text { Debregeasia longifolia } \\
\text { (Burm.f.) Wedd. }\end{array}$ & $\begin{array}{l}\text { Mao ma } \\
\text { qiang ga }\end{array}$ & Shrub & Urticaceae & Fruit & Ripe fruits are eaten fresh & $\begin{array}{l}\text { Lonajia } \\
\text { Village, Jiayin } \\
\text { Township }\end{array}$ & $\begin{array}{l}201,506- \\
31\end{array}$ & 2.1 & 0.51 \\
\hline $\begin{array}{l}\text { Debregeasia orientalis C. J. } \\
\text { Chen }\end{array}$ & O ce bu & Shrub & Urticaceae & $\begin{array}{l}\text { Fruit, } \\
\text { leaf, } \\
\text { tender } \\
\text { stem }\end{array}$ & $\begin{array}{l}\text { Leaf and stem: potherb; } \\
\text { fruit: eaten fresh }\end{array}$ & $\begin{array}{l}\text { Baohua } \\
\text { Township }\end{array}$ & HHD-19 & 2.5 & 0.55 \\
\hline $\begin{array}{l}\text { Elatostema involucratum } \\
\text { Franch. and Sav. }\end{array}$ & $\begin{array}{l}\text { Luo bu. a } \\
\text { bo }\end{array}$ & Herb & Urticaceae & $\begin{array}{l}\text { Tender } \\
\text { stem, } \\
\text { leaf }\end{array}$ & Potherb & $\begin{array}{l}\text { Niujiaozhai } \\
\text { Township }\end{array}$ & $\begin{array}{l}201,606- \\
13\end{array}$ & 1.9 & 0.40 \\
\hline $\begin{array}{l}\text { Gonostegia hirta (Blume ex } \\
\text { Hassk.) Miq. }\end{array}$ & $\begin{array}{l}\text { Pa qian a } \\
\text { bo }\end{array}$ & Herb & Urticaceae & $\begin{array}{l}\text { Tender } \\
\text { stem, } \\
\text { leaf }\end{array}$ & Potherb & $\begin{array}{l}\text { Lüchun } \\
\text { County }\end{array}$ & LB-22 & 2.1 & 0.54 \\
\hline $\begin{array}{l}\text { Lecanthus peduncularis (Wall. } \\
\text { ex Royle) Wedd. }\end{array}$ & $\begin{array}{l}\text { A che pa } \\
\text { nv }\end{array}$ & Herb & Urticaceae & $\begin{array}{l}\text { Whole } \\
\text { plant }\end{array}$ & $\begin{array}{l}\text { Potherb (usually made } \\
\text { into soup) }\end{array}$ & $\begin{array}{l}\text { Baohua } \\
\text { Township }\end{array}$ & HHD-30 & 2.2 & 0.48 \\
\hline $\begin{array}{l}\text { Castanopsis calathiformis } \\
\text { (Skan) Rehder and E.H.Wilson }\end{array}$ & A ba a bo & Tree & Fagaceae & Seed & $\begin{array}{l}\text { Roasted and eaten } \\
\text { (kernel) }\end{array}$ & $\begin{array}{l}\text { Baohua } \\
\text { Township }\end{array}$ & HHD-20 & 0.8 & 0.18 \\
\hline $\begin{array}{l}\text { Castanopsis carlesii var. } \\
\text { spinulosa W.C.Cheng and } \\
\text { C.S.Chao }\end{array}$ & $\begin{array}{l}\text { Che gian a } \\
\text { bo }\end{array}$ & Tree & Fagaceae & Seed & $\begin{array}{l}\text { Roasted and eaten } \\
\text { (kernel) }\end{array}$ & $\begin{array}{l}\text { Niujiaozhai } \\
\text { Township }\end{array}$ & $\begin{array}{l}201,606- \\
10\end{array}$ & 0.5 & 0.17 \\
\hline $\begin{array}{l}\text { Castanopsis indica (Roxb. ex } \\
\text { Lindl.) A.DC. }\end{array}$ & $\begin{array}{l}\text { Che si a } \\
\text { bo }\end{array}$ & Tree & Fagaceae & Seed & $\begin{array}{l}\text { Roasted and eaten } \\
\text { (kernel) }\end{array}$ & $\begin{array}{l}\text { Lüchun } \\
\text { County }\end{array}$ & LB-21 & 0.8 & 0.05 \\
\hline $\begin{array}{l}\text { Castanopsis mekongensis } \\
\text { A.Camus }\end{array}$ & & Tree & Fagaceae & Seed & $\begin{array}{l}\text { Roasted and eaten } \\
\text { (kernel) }\end{array}$ & $\begin{array}{l}\text { Shuiyan } \\
\text { Village, } \\
\text { Ma'andi } \\
\text { Township }\end{array}$ & $\begin{array}{l}201,511- \\
24\end{array}$ & 0.7 & 0.20 \\
\hline
\end{tabular}


Table 3 Inventory of WEP species in Honghe terraced rice paddy system (Continued)

\begin{tabular}{|c|c|c|c|c|c|c|c|c|c|}
\hline Scientific name & $\begin{array}{l}\text { Vernacular } \\
\text { Name }\end{array}$ & $\begin{array}{l}\text { Life } \\
\text { form }\end{array}$ & Family name & $\begin{array}{l}\text { Parts } \\
\text { used }\end{array}$ & Preparation and uses & Study sites & $\begin{array}{l}\text { Voucher } \\
\text { number }\end{array}$ & FUI & UV \\
\hline $\begin{array}{l}\text { Lithocarpus megalophyllus } \\
\text { Rehder and E.H.Wilson }\end{array}$ & A biu a bo & Tree & Fagaceae & Fruit & Ripe fruits are eaten fresh & $\begin{array}{l}\text { Baohua } \\
\text { Township }\end{array}$ & $\begin{array}{l}201,511- \\
34\end{array}$ & 2.8 & 0.46 \\
\hline $\begin{array}{l}\text { Myricae esculenta Buch.-Ham. } \\
\text { ex D. Don }\end{array}$ & & Tree & Myricaceae & Fruit & $\begin{array}{l}\text { Fruits eaten fresh or } \\
\text { liquor brewing }\end{array}$ & $\begin{array}{l}\text { Niujiaozhai } \\
\text { Township }\end{array}$ & $\begin{array}{l}201,606- \\
06\end{array}$ & 2.0 & 0.41 \\
\hline $\begin{array}{l}\text { Gynostemma pubescens } \\
\text { (Gagnep.) C.Y.Wu }\end{array}$ & $\begin{array}{l}\text { Ka kui zha } \\
\text { ha }\end{array}$ & Herb & Cucurbitaceae & $\begin{array}{l}\text { Leaf, } \\
\text { tender } \\
\text { stem }\end{array}$ & Tea substitute & $\begin{array}{l}\text { Lonajia } \\
\text { Village, Jiayin } \\
\text { Township }\end{array}$ & $\begin{array}{l}201,506- \\
44\end{array}$ & 0.2 & 0.18 \\
\hline $\begin{array}{l}\text { Hemsleya macrosperma } \\
\text { C.Y.Wu }\end{array}$ & A za ku xi & Herb & Cucurbitaceae & $\begin{array}{l}\text { Tender } \\
\text { leaf }\end{array}$ & Potherb & $\begin{array}{l}\text { Xiongjia } \\
\text { Village, } \\
\text { Adebo } \\
\text { Township }\end{array}$ & $\begin{array}{l}201,506- \\
02\end{array}$ & 2.5 & 0.40 \\
\hline $\begin{array}{l}\text { Hodgsonia macrocarpa } \\
\text { (Blume) Cogn. }\end{array}$ & $\begin{array}{l}\text { Zha qi gu } \\
\text { lu }\end{array}$ & Liana & Cucurbitaceae & Seed & $\begin{array}{l}\text { Eaten directly, or used for } \\
\text { pressing oil }\end{array}$ & $\begin{array}{l}\text { Shuiyan } \\
\text { Village, } \\
\text { Ma'andi } \\
\text { Township }\end{array}$ & $\begin{array}{l}201,511- \\
02\end{array}$ & 1.9 & 0.48 \\
\hline $\begin{array}{l}\text { Momordica cochinchinensis } \\
\text { (Lour.) Spreng. }\end{array}$ & Bei ba na & Liana & Cucurbitaceae & $\begin{array}{l}\text { Tender } \\
\text { stem, } \\
\text { leaf }\end{array}$ & Potherb & $\begin{array}{l}\text { Shuiyan } \\
\text { Village, } \\
\text { Ma'andi } \\
\text { Township }\end{array}$ & $\begin{array}{l}201,511- \\
30\end{array}$ & 2.9 & 0.44 \\
\hline Salacia sessiliflora Hand.-Mazz. & $\begin{array}{l}\text { A ka la ma } \\
\text { a bo }\end{array}$ & Shrub & Celastraceae & Fruit & Ripe fruits are eaten fresh & $\begin{array}{l}\text { Lüchun } \\
\text { County }\end{array}$ & $\begin{array}{l}201,606- \\
25\end{array}$ & 2.6 & 0.41 \\
\hline Oxalis corniculata L. & Suan ji cao & Herb & Oxalidaceae & $\begin{array}{l}\text { Stem, } \\
\text { leaf }\end{array}$ & $\begin{array}{l}\text { Potherb: blanched in } \\
\text { boiled water, then } \\
\text { soaked in cold water for } \\
2 \mathrm{~h}\end{array}$ & $\begin{array}{l}\text { Niujiaozhai } \\
\text { Township }\end{array}$ & $\begin{array}{l}201,606- \\
01\end{array}$ & 2.3 & 0.47 \\
\hline $\begin{array}{l}\text { Elaeocarpus decipiens } \\
\text { F.B.Forbes and Hemsl. }\end{array}$ & Na ci ci ha & Tree & Elaeocarpaceae & Fruit & Ripe fruits are eaten fresh & $\begin{array}{l}\text { Shuiyan } \\
\text { Village, } \\
\text { Ma'andi } \\
\text { Township }\end{array}$ & $\begin{array}{l}201,511- \\
08\end{array}$ & 1.9 & 0.58 \\
\hline $\begin{array}{l}\text { Garcinia cowa Roxb. ex } \\
\text { Choisy }\end{array}$ & $\begin{array}{l}\text { Huang xin } \\
\text { shu }\end{array}$ & Tree & Clusiaceae & Fruit & Ripe fruits are eaten fresh & $\begin{array}{l}\text { Baohua } \\
\text { Township }\end{array}$ & $\begin{array}{l}201,511- \\
35\end{array}$ & 2.0 & 0.47 \\
\hline $\begin{array}{l}\text { Garcinia multiflora Champ. ex } \\
\text { Benth. }\end{array}$ & $\begin{array}{l}\text { Qiu guo a } \\
\text { si }\end{array}$ & Tree & Clusiaceae & Fruit & Ripe fruits are eaten fresh & $\begin{array}{l}\text { Niujiaozhai } \\
\text { Township }\end{array}$ & $\begin{array}{l}201,606- \\
02\end{array}$ & 2.4 & 0.42 \\
\hline $\begin{array}{l}\text { Garcinia xanthochymus } \\
\text { Hook.f. ex T.Anderson }\end{array}$ & $\begin{array}{l}\text { A bu. bu. } \\
\text { qie }\end{array}$ & Tree & Clusiaceae & Fruit & Ripe fruits are eaten fresh & $\begin{array}{l}\text { Niujiaozhai } \\
\text { Township }\end{array}$ & $\begin{array}{l}201,606- \\
04\end{array}$ & 2.1 & 0.52 \\
\hline $\begin{array}{l}\text { Cratoxylum cochinchinense } \\
\text { (Lour.) Blume }\end{array}$ & $\begin{array}{l}\text { Jiu ge ge } \\
\text { qia }\end{array}$ & Tree & Hypericaceae & $\begin{array}{l}\text { Tender } \\
\text { leaf, } \\
\text { young } \\
\text { fruit }\end{array}$ & $\begin{array}{l}\text { Tender leaves: tea } \\
\text { substitute; young fruit: } \\
\text { flavoring agent }\end{array}$ & $\begin{array}{l}\text { Niujiaozhai } \\
\text { Township }\end{array}$ & $\begin{array}{l}201,606- \\
11\end{array}$ & 2.2 & 0.49 \\
\hline $\begin{array}{l}\text { Cratoxylum formosum subsp. } \\
\text { pruniflorum (Kurz) Gogelein }\end{array}$ & A on a bo & Tree & Hypericaceae & $\begin{array}{l}\text { Tender } \\
\text { leaf }\end{array}$ & Tea substitute & $\begin{array}{l}\text { Lagu Village, } \\
\text { Sanmeng } \\
\text { Township }\end{array}$ & HHD-44 & 0.9 & 0.25 \\
\hline $\begin{array}{l}\text { Curculigo capitulata (Lour.) } \\
\text { Kuntze }\end{array}$ & $\begin{array}{l}\text { Ma ni zu } \\
\text { se }\end{array}$ & Herb & Hypoxidaceae & $\begin{array}{l}\text { Fruit, } \\
\text { tender } \\
\text { leaf, } \\
\text { tender } \\
\text { stem }\end{array}$ & $\begin{array}{l}\text { Fruit: eaten fresh; leaves } \\
\text { and stem: potherb }\end{array}$ & $\begin{array}{l}\text { Baohua } \\
\text { Township }\end{array}$ & HHD-18 & 2.0 & 0.56 \\
\hline Curculigo sinensis S. C. Chen & $\begin{array}{l}\text { Mei la pa } \\
\text { jia }\end{array}$ & Herb & Hypoxidaceae & Fruit & Ripe fruits are eaten fresh & $\begin{array}{l}\text { Baohua } \\
\text { Township }\end{array}$ & $\begin{array}{l}201,511- \\
36\end{array}$ & 1.9 & 0.56 \\
\hline Passiflora wilsonii Hemsl. & Ba ze & Liana & Passifloraceae & Fruit & Ripe fruits are eaten fresh & $\begin{array}{l}\text { Shuiyan } \\
\text { Village, } \\
\text { Ma'andi } \\
\text { Township }\end{array}$ & $\begin{array}{l}201,511- \\
04\end{array}$ & 2.5 & 0.51 \\
\hline Flacourtia ramontchi L'Hér. & $\begin{array}{l}\text { A zi long } \\
\text { jie a bo }\end{array}$ & Shrub & Salicaceae & Fruit & $\begin{array}{l}\text { Ripe fruits are eaten } \\
\text { fresh, or made into jam, } \\
\text { or preserved }\end{array}$ & $\begin{array}{l}\text { Lüshuge } \\
\text { Village, Jiayin } \\
\text { Township }\end{array}$ & $\begin{array}{l}201,610- \\
10\end{array}$ & 2.4 & 0.58 \\
\hline Baccaurea ramiflora Lour. & Si suo a si & Tree & Phyllanthaceae & Fruit & Ripe fruits are eaten fresh & Lonajia & $201,506-$ & 4.2 & 0.89 \\
\hline
\end{tabular}


Table 3 Inventory of WEP species in Honghe terraced rice paddy system (Continued)

\begin{tabular}{|c|c|c|c|c|c|c|c|c|c|}
\hline Scientific name & $\begin{array}{l}\text { Vernacular } \\
\text { Name }\end{array}$ & $\begin{array}{l}\text { Life } \\
\text { form }\end{array}$ & Family name & $\begin{array}{l}\text { Parts } \\
\text { used }\end{array}$ & Preparation and uses & Study sites & $\begin{array}{l}\text { Voucher } \\
\text { number }\end{array}$ & FUI & UV \\
\hline & & & & & & $\begin{array}{l}\text { Village, Jiayin } \\
\text { Township }\end{array}$ & 34 & & \\
\hline Phyllanthus emblica L. & $\begin{array}{l}\text { Bo can xi } \\
\text { ka, xi qia } \\
\text { ha }\end{array}$ & Tree & Phyllanthaceae & $\begin{array}{l}\text { Bark, } \\
\text { fruit }\end{array}$ & $\begin{array}{l}\text { Fruit: eaten fresh; bark: } \\
\text { scraching off the inside } \\
\text { tender bark to make } \\
\text { dishes }\end{array}$ & $\begin{array}{l}\text { Lüchun } \\
\text { County }\end{array}$ & $\begin{array}{l}201,606- \\
29\end{array}$ & 4.9 & 0.90 \\
\hline Rotala indica (Willd.) Koehne & En ni a bo & Herb & Lythraceae & $\begin{array}{l}\text { Tender } \\
\text { shoot }\end{array}$ & Potherb & $\begin{array}{l}\text { Lüshuge } \\
\text { Village, Jiayin } \\
\text { Township }\end{array}$ & $\begin{array}{l}201,610- \\
16\end{array}$ & 2.2 & 0.42 \\
\hline $\begin{array}{l}\text { Rotala rotundifolia (Buch.- } \\
\text { Ham. ex Roxb.) Koehne }\end{array}$ & & Herb & Lythraceae & $\begin{array}{l}\text { Tender } \\
\text { leaf, } \\
\text { tender } \\
\text { stem }\end{array}$ & Potherb & $\begin{array}{l}\text { Baohua } \\
\text { Township }\end{array}$ & HHD-27 & 2.1 & 0.52 \\
\hline $\begin{array}{l}\text { Cleistocalyx operculatus } \\
\text { (Roxb.) Merr. and L.M.Perry }\end{array}$ & & Tree & Myrtaceae & Fruit & Ripe fruits are eaten fresh & $\begin{array}{l}\text { Lonajia } \\
\text { Village, Jiayin } \\
\text { Township }\end{array}$ & $\begin{array}{l}201,506- \\
27\end{array}$ & 1.6 & 0.56 \\
\hline $\begin{array}{l}\text { Decaspermum parviflorum } \\
\text { (Lam.) A.J.Scott }\end{array}$ & $\begin{array}{l}\text { A gong } \\
\text { gong ni a } \\
\text { bo }\end{array}$ & Tree & Myrtaceae & Fruit & Ripe fruits are eaten fresh & $\begin{array}{l}\text { Lonajia } \\
\text { Village, Jiayin } \\
\text { Township }\end{array}$ & $\begin{array}{l}201,506- \\
28\end{array}$ & 2.4 & 0.38 \\
\hline $\begin{array}{l}\text { Syzygium fluviatile (Hemsl.) } \\
\text { Merr. and L.M.Perry }\end{array}$ & $\begin{array}{l}\text { Me ran me } \\
\text { xiu na ci a } \\
\text { bo }\end{array}$ & Shrub & Myrtaceae & Fruit & Ripe fruits are eaten fresh & $\begin{array}{l}\text { The junction } \\
\text { of Lüchun } \\
\text { County and } \\
\text { Yuanyang } \\
\text { County }\end{array}$ & $\begin{array}{l}201,506- \\
09\end{array}$ & 1.6 & 0.49 \\
\hline $\begin{array}{l}\text { Syzygium yunnanense Merr. } \\
\text { and L.M.Perry }\end{array}$ & O ho & Tree & Myrtaceae & Fruit & Ripe fruits are eaten fresh & $\begin{array}{l}\text { Lüchun } \\
\text { County }\end{array}$ & $\begin{array}{l}201,606- \\
24\end{array}$ & 2.8 & 0.45 \\
\hline $\begin{array}{l}\text { Medinilla radiciflora C.Y.Wu ex } \\
\text { C.Chen }\end{array}$ & & Shrub & Melastomataceae & Fruit & Ripe fruits are eaten fresh & $\begin{array}{l}\text { Shuiyan } \\
\text { Village, } \\
\text { Ma'andi } \\
\text { Township }\end{array}$ & $\begin{array}{l}201,511- \\
03\end{array}$ & 2.4 & 0.39 \\
\hline $\begin{array}{l}\text { Medinilla septentrionalis (W.W. } \\
\text { Sm.) H.L. Li }\end{array}$ & $\begin{array}{l}\text { Qian ben } \\
\text { er a si }\end{array}$ & Shrub & Melastomataceae & Fruit & Ripe fruits are eaten fresh & $\begin{array}{l}\text { Niujiaozhai } \\
\text { Township }\end{array}$ & $\begin{array}{l}201,606- \\
05\end{array}$ & 2.8 & 0.48 \\
\hline Melastoma affine D. Don & Bei bai & Shrub & Melastomataceae & Fruit & Ripe fruits are eaten fresh & $\begin{array}{l}\text { Xiaoxinjie } \\
\text { Township }\end{array}$ & LB-04 & 2.2 & 0.46 \\
\hline Melastoma normale D. Don. & $\begin{array}{l}\text { Yang er ba } \\
\text { cui }\end{array}$ & Shrub & Melastomataceae & $\begin{array}{l}\text { Fruit, } \\
\text { leaf }\end{array}$ & Ripe fruits are eaten fresh & $\begin{array}{l}\text { Lüchun } \\
\text { County }\end{array}$ & $\begin{array}{l}201,606- \\
20\end{array}$ & 2.6 & 0.47 \\
\hline $\begin{array}{l}\text { Osbeckia opipara C.Y. Wu et } \\
\text { C. Chen }\end{array}$ & Bi ji & Shrub & Melastomataceae & $\begin{array}{l}\text { Root, } \\
\text { stem }\end{array}$ & $\begin{array}{l}\text { Potherb (usually stewed } \\
\text { with meat) }\end{array}$ & $\begin{array}{l}\text { The junction } \\
\text { of Lüchun } \\
\text { County and } \\
\text { Yuanyang } \\
\text { County }\end{array}$ & $\begin{array}{l}201,506- \\
11\end{array}$ & 2.2 & 0.57 \\
\hline Canarium album (Lour.) DC. & Bei le a si & Tree & Burseraceae & Fruit & $\begin{array}{l}\text { Ripe fruits are eaten } \\
\text { fresh, or preserved }\end{array}$ & $\begin{array}{l}\text { Shuiyan } \\
\text { Village, } \\
\text { Ma'andi } \\
\text { Township }\end{array}$ & $\begin{array}{l}201,511- \\
10\end{array}$ & 2.3 & 0.40 \\
\hline Canarium pimela K.D.Koenig & Si mo a si & Tree & Burseraceae & Fruit & $\begin{array}{l}\text { Ripe fruits are eaten } \\
\text { fresh, or preserved }\end{array}$ & $\begin{array}{l}\text { Niujiaozhai } \\
\text { Township }\end{array}$ & $\begin{array}{l}201,606- \\
07\end{array}$ & 2.4 & 0.44 \\
\hline Canarium strictum Roxb. & $\begin{array}{l}\text { A bo ma } \\
\text { dai }\end{array}$ & Tree & Burseraceae & Fruit & $\begin{array}{l}\text { Ripe fruits are eaten } \\
\text { fresh, or preserved }\end{array}$ & $\begin{array}{l}\text { Baohua } \\
\text { Township }\end{array}$ & $\begin{array}{l}201,511- \\
42\end{array}$ & 2.5 & 0.46 \\
\hline $\begin{array}{l}\text { Choerospondias axillaris } \\
\text { (Roxb.) B. L. Burtt and A. W. } \\
\text { Hill }\end{array}$ & $\begin{array}{l}\text { Gei ha a } \\
\text { bo }\end{array}$ & Tree & Anacardiaceae & Fruit & $\begin{array}{l}\text { Fruits eaten fresh or } \\
\text { liquor brewing }\end{array}$ & $\begin{array}{l}\text { Baohua } \\
\text { Township }\end{array}$ & $\begin{array}{l}201,511- \\
41\end{array}$ & 2.2 & 0.55 \\
\hline $\begin{array}{l}\text { Dracontomelon duperreanum } \\
\text { Pierre }\end{array}$ & A zi ren a & Tree & Anacardiaceae & Fruit & $\begin{array}{l}\text { Ripe fruits are eaten } \\
\text { fresh, or preserved }\end{array}$ & $\begin{array}{l}\text { Lagu Village, } \\
\text { Sanmeng } \\
\text { Township }\end{array}$ & HHD-37 & 2.3 & 0.49 \\
\hline Mangifera sylvatica Roxb. & & Tree & Anacardiaceae & Fruit & Ripe fruits are eaten fresh & $\begin{array}{l}\text { Qingkou } \\
\text { Village, Xinjie }\end{array}$ & $\begin{array}{l}201,506- \\
14\end{array}$ & 2.0 & 0.40 \\
\hline
\end{tabular}


Table 3 Inventory of WEP species in Honghe terraced rice paddy system (Continued)

\begin{tabular}{|c|c|c|c|c|c|c|c|c|c|}
\hline Scientific name & $\begin{array}{l}\text { Vernacular } \\
\text { Name }\end{array}$ & $\begin{array}{l}\text { Life } \\
\text { form }\end{array}$ & Family name & $\begin{array}{l}\text { Parts } \\
\text { used }\end{array}$ & Preparation and uses & Study sites & $\begin{array}{l}\text { Voucher } \\
\text { number }\end{array}$ & FUI & UV \\
\hline & & & & & & Township & & & \\
\hline Rhus chinensis Mill. & $\begin{array}{l}\text { Ha da da } \\
\text { xiu }\end{array}$ & Tree & Anacardiaceae & Fruit & Preserved fruit & $\begin{array}{l}\text { Lonajia } \\
\text { Village, Jiayin } \\
\text { Township }\end{array}$ & $\begin{array}{l}201,506- \\
42\end{array}$ & 2.8 & 0.53 \\
\hline Arytera littoralis Blume & $\begin{array}{l}\text { Ta mo si } \\
\text { song }\end{array}$ & Tree & Sapindaceae & Shoot & Potherb & $\begin{array}{l}\text { Lonajia } \\
\text { Village, Jiayin } \\
\text { Township }\end{array}$ & $\begin{array}{l}201,506- \\
48\end{array}$ & 2.6 & 0.52 \\
\hline $\begin{array}{l}\text { Acronychia pedunculata (L.) } \\
\text { Miq. }\end{array}$ & & Tree & Rutaceae & Fruit & Flavoring agent & $\begin{array}{l}\text { Lüshuge } \\
\text { Village, Jiayin } \\
\text { Township }\end{array}$ & $\begin{array}{l}201,610- \\
11\end{array}$ & 2.3 & 0.43 \\
\hline $\begin{array}{l}\text { Tetradium austrosinense } \\
\text { (Hand.-Mazz.) Hartley }\end{array}$ & & Tree & Rutaceae & Fruit & $\begin{array}{l}\text { Fruits are edible and } \\
\text { used for pressing oil }\end{array}$ & $\begin{array}{l}\text { Xiaoxinjie } \\
\text { Township }\end{array}$ & $\begin{array}{l}201,506- \\
07\end{array}$ & 1.6 & 0.47 \\
\hline $\begin{array}{l}\text { Zanthoxylum bungeanum } \\
\text { Maxim. }\end{array}$ & A zao & Tree & Rutaceae & Fruit & Flavoring agent & $\begin{array}{l}\text { Xiongjia } \\
\text { Village, } \\
\text { Adebo } \\
\text { Township }\end{array}$ & $\begin{array}{l}\text { HHD- } \\
013\end{array}$ & 2.4 & 0.48 \\
\hline Zanthoxylum scandens Blume & & Shrub & Rutaceae & Fruit & Flavoring agent & $\begin{array}{l}\text { Lonajia } \\
\text { Village, Jiayin } \\
\text { Township }\end{array}$ & $\begin{array}{l}201,506- \\
38\end{array}$ & 2.3 & 0.43 \\
\hline Zanthoxylum simulans Hance & & Shrub & Rutaceae & Fruit & Flavoring agent & $\begin{array}{l}\text { Lagu Village, } \\
\text { Sanmeng } \\
\text { Township }\end{array}$ & HHD-38 & 2.3 & 0.44 \\
\hline $\begin{array}{l}\text { Ailanthus altissima (Mill.) } \\
\text { Swingle }\end{array}$ & $\begin{array}{l}\text { Qi la wu } \\
\text { ha }\end{array}$ & Tree & Simaroubaceae & $\begin{array}{l}\text { Tender } \\
\text { leaf }\end{array}$ & Potherb & $\begin{array}{l}\text { Lonajia } \\
\text { Village, Jiayin } \\
\text { Township }\end{array}$ & $\begin{array}{l}201,506- \\
50\end{array}$ & 1.8 & 0.41 \\
\hline Bombax ceiba L. & & Tree & Malvaceae & Flower & Potherb & $\begin{array}{l}\text { Lagu Village, } \\
\text { Sanmeng } \\
\text { Township }\end{array}$ & HHD-49 & 2.2 & 0.42 \\
\hline $\begin{array}{l}\text { Microcos nervosa (Lour.) S.Y. } \\
\mathrm{Hu}\end{array}$ & & Tree & Malvaceae & Fruit & Ripe fruits are eaten fresh & $\begin{array}{l}\text { Baohua } \\
\text { Township }\end{array}$ & $\begin{array}{l}201,511- \\
38\end{array}$ & 2.3 & 0.45 \\
\hline Sterculia brevissima H.H.Hsue & $\begin{array}{l}\text { Sa qiu huo } \\
\text { bi }\end{array}$ & Shrub & Malvaceae & Seed & $\begin{array}{l}\text { Roasted and eaten } \\
\text { (kernel) }\end{array}$ & $\begin{array}{l}\text { Xiongjia } \\
\text { Village, } \\
\text { Adebo } \\
\text { Township }\end{array}$ & $\begin{array}{l}\text { HHD- } \\
017\end{array}$ & 0.1 & 0.21 \\
\hline Sterculia lanceolata Cav. & $\begin{array}{l}\text { Sa qiu huo } \\
\text { bi }\end{array}$ & Tree & Malvaceae & Seed & $\begin{array}{l}\text { Roasted and eaten } \\
\text { (kernel) }\end{array}$ & $\begin{array}{l}\text { Xiongjia } \\
\text { Village, } \\
\text { Adebo } \\
\text { Township }\end{array}$ & $\begin{array}{l}\text { HHD- } \\
016\end{array}$ & 0.1 & 0.16 \\
\hline Sterculia pexa Pierre & $\begin{array}{l}\text { Ni hei gei } \\
\text { zi a bo }\end{array}$ & Tree & Malvaceae & Seed & Stir-fried & $\begin{array}{l}\text { Lagu Village, } \\
\text { Sanmeng } \\
\text { Township }\end{array}$ & HHD-41 & 2.5 & 0.56 \\
\hline Capparis masaikai H. Lév. & & Liana & Capparaceae & Seed & Natural sweetener & $\begin{array}{l}\text { Qingkou } \\
\text { Village, Xinjie } \\
\text { Township }\end{array}$ & $\begin{array}{l}201,506- \\
20\end{array}$ & 0.8 & 0.2 \\
\hline $\begin{array}{l}\text { Crateva unilocularis Buch.- } \\
\text { Ham. }\end{array}$ & $\begin{array}{l}\text { Man nei } \\
\text { luo ba }\end{array}$ & Tree & Capparaceae & $\begin{array}{l}\text { Tender } \\
\text { stem, } \\
\text { leaf }\end{array}$ & $\begin{array}{l}\text { Made into pickles } \\
\text { (preserved) }\end{array}$ & $\begin{array}{l}\text { Lüshuge } \\
\text { Village, Jiayin } \\
\text { Township }\end{array}$ & $\begin{array}{l}201,610- \\
14\end{array}$ & 3.9 & 0.75 \\
\hline Stixis suaveolens (Roxb.) Pierre & & Liana & Capparaceae & $\begin{array}{l}\text { Fruit, } \\
\text { tender } \\
\text { leaf }\end{array}$ & $\begin{array}{l}\text { Fruit: eaten fresh; tender } \\
\text { leaves: tea substitute }\end{array}$ & $\begin{array}{l}\text { Lüchun } \\
\text { County }\end{array}$ & $\begin{array}{l}201,606- \\
30\end{array}$ & 2.2 & 0.42 \\
\hline $\begin{array}{l}\text { Capsella bursa-pastoris (L.) } \\
\text { Medik. }\end{array}$ & A zu o qi & Herb & Brassicaceae & $\begin{array}{l}\text { Tender } \\
\text { stem, } \\
\text { leaf }\end{array}$ & Potherb & $\begin{array}{l}\text { Lü shuge } \\
\text { Village, Jiayin } \\
\text { Township }\end{array}$ & $\begin{array}{l}201,610- \\
19\end{array}$ & 2.7 & 0.45 \\
\hline $\begin{array}{l}\text { Gynostemma pentaphyllum } \\
\text { (Thunb.) Makino }\end{array}$ & & Herb & Brassicaceae & $\begin{array}{l}\text { Tender } \\
\text { stem } \\
\text { and } \\
\text { leaves }\end{array}$ & Potherb or tea substitute & $\begin{array}{l}\text { Lonajia } \\
\text { Village, Jiayin } \\
\text { Township }\end{array}$ & $\begin{array}{l}201,506- \\
46\end{array}$ & 2.0 & 0.49 \\
\hline
\end{tabular}


Table 3 Inventory of WEP species in Honghe terraced rice paddy system (Continued)

\begin{tabular}{|c|c|c|c|c|c|c|c|c|c|}
\hline Scientific name & $\begin{array}{l}\text { Vernacular } \\
\text { Name }\end{array}$ & $\begin{array}{l}\text { Life } \\
\text { form }\end{array}$ & Family name & $\begin{array}{l}\text { Parts } \\
\text { used }\end{array}$ & Preparation and uses & Study sites & $\begin{array}{l}\text { Voucher } \\
\text { number }\end{array}$ & FUI & UV \\
\hline Nasturtium officinale R.Br. & Xi yang cai & Herb & Brassicaceae & $\begin{array}{l}\text { Tender } \\
\text { leaf }\end{array}$ & Potherb & $\begin{array}{l}\text { Xiaoxinjie } \\
\text { Township }\end{array}$ & LB-10 & 3.4 & 0.66 \\
\hline $\begin{array}{l}\text { Rorippa islandica (Oeder) } \\
\text { Borbás }\end{array}$ & & Herb & Brassicaceae & $\begin{array}{l}\text { Tender } \\
\text { leaf }\end{array}$ & $\begin{array}{l}\text { Potherb (boiled for 5-10 } \\
\text { min, then soaked in } \\
\text { water to remove } \\
\text { pungent taste) }\end{array}$ & $\begin{array}{l}\text { Lagu Village, } \\
\text { Sanmeng } \\
\text { Township }\end{array}$ & HHD-53 & 2.4 & 0.50 \\
\hline Erythropalum scandens Blume & $\begin{array}{l}\text { Ha jia ha } \\
\text { na bei } \\
\text { ying }\end{array}$ & Liana & Olacaceae & $\begin{array}{l}\text { Tender } \\
\text { stem, } \\
\text { leaf }\end{array}$ & Potherb & $\begin{array}{l}\text { Xiaoxinjie } \\
\text { Township }\end{array}$ & LB-15 & 2.1 & 0.52 \\
\hline $\begin{array}{l}\text { Korthalsella japonica (Thunb.) } \\
\text { Engl. }\end{array}$ & De la & Shrub & Santalaceae & Fruit & Ripe fruits are eaten fresh & $\begin{array}{l}\text { Lonajia } \\
\text { Village, Jiayin } \\
\text { Township }\end{array}$ & $\begin{array}{l}201,506- \\
33\end{array}$ & 1.7 & 0.39 \\
\hline Pyrularia edulis (Wall.) A. DC. & $\begin{array}{l}\text { A ke ke } \\
\text { ran a si }\end{array}$ & Tree & Santalaceae & Fruit & $\begin{array}{l}\text { Ripe fruits are stewed or } \\
\text { stir-fried }\end{array}$ & $\begin{array}{l}\text { Niujiaozhai } \\
\text { Township }\end{array}$ & $\begin{array}{l}201,606- \\
12\end{array}$ & 2.3 & 0.50 \\
\hline $\begin{array}{l}\text { Myosoton aquaticum (L.) } \\
\text { Moench }\end{array}$ & $\begin{array}{l}\text { Qian chu a } \\
\text { ma }\end{array}$ & Herb & Caryophyllaceae & $\begin{array}{l}\text { Tender } \\
\text { leaf, } \\
\text { tender } \\
\text { stem }\end{array}$ & Potherb & $\begin{array}{l}\text { Xiaoxinjie } \\
\text { Township }\end{array}$ & LB-11 & 2.2 & 0.46 \\
\hline Amaranthus spinosus $\mathrm{L}$. & $\begin{array}{l}\text { Wo zu wo } \\
\text { niu }\end{array}$ & Herb & Amaranthaceae & $\begin{array}{l}\text { Tender } \\
\text { leaf, } \\
\text { tender } \\
\text { stem }\end{array}$ & Potherb & $\begin{array}{l}\text { Qingkou } \\
\text { Village, Xinjie } \\
\text { Township }\end{array}$ & $\begin{array}{l}201,506- \\
21\end{array}$ & 2.5 & 0.53 \\
\hline Amaranthus lividus L. & & Herb & Amaranthaceae & $\begin{array}{l}\text { Leaf, } \\
\text { stem }\end{array}$ & Potherb & $\begin{array}{l}\text { Lagu Village, } \\
\text { Sanmeng } \\
\text { Township }\end{array}$ & HHD-52 & 2.2 & 0.47 \\
\hline Amaranthus viridis $\mathrm{L}$. & $\begin{array}{l}\text { La huo pa } \\
\text { ni }\end{array}$ & Herb & Amaranthaceae & $\begin{array}{l}\text { Tender } \\
\text { leaf, } \\
\text { tender } \\
\text { stem }\end{array}$ & Potherb & $\begin{array}{l}\text { Lüshuge } \\
\text { Village, Jiayin } \\
\text { Township }\end{array}$ & $\begin{array}{l}201,610- \\
23\end{array}$ & 3.6 & 0.61 \\
\hline Chenopodium album $\mathrm{L}$. & $\begin{array}{l}\text { Ge xia wo } \\
\text { niu }\end{array}$ & Herb & Amaranthaceae & Shoot & Potherb & $\begin{array}{l}\text { Baohua } \\
\text { Township }\end{array}$ & HHD-28 & 2.3 & 0.49 \\
\hline Phytolacca acinosa Roxb & Kan bo & Herb & Phytolaccaceae & Leaf & Potherb & $\begin{array}{l}\text { Qingkou } \\
\text { Village, Xinjie } \\
\text { Township }\end{array}$ & $\begin{array}{l}201,506- \\
22\end{array}$ & 2.3 & 0.44 \\
\hline Portulaca oleracea L. & $\begin{array}{l}\text { Yi ca mo } \\
\text { ni }\end{array}$ & Herb & Portulacaceae & $\begin{array}{l}\text { Tender } \\
\text { leaf, } \\
\text { tender } \\
\text { stem }\end{array}$ & Potherb & $\begin{array}{l}\text { Lagu Village, } \\
\text { Sanmeng } \\
\text { Township }\end{array}$ & HHD-51 & 1.8 & 0.52 \\
\hline $\begin{array}{l}\text { Dendrobenthamia } \\
\text { hongkongensis (Hemsl.) } \\
\text { Hutch. }\end{array}$ & & Tree & Cornaceae & Fruit & $\begin{array}{l}\text { Fruits eaten fresh or } \\
\text { liquor brewing }\end{array}$ & $\begin{array}{l}\text { Baohua } \\
\text { Township }\end{array}$ & $\begin{array}{l}201,511- \\
39\end{array}$ & 1.9 & 0.56 \\
\hline $\begin{array}{l}\text { Dendrobenthamia } \\
\text { melanotricha (Pojark.) } \\
\text { W.P.Fang }\end{array}$ & & Tree & Cornaceae & Fruit & Ripe fruits are eaten fresh & $\begin{array}{l}\text { Shuiyan } \\
\text { Village, } \\
\text { Ma'andi } \\
\text { Township }\end{array}$ & $\begin{array}{l}201,511- \\
06\end{array}$ & 2.5 & 0.47 \\
\hline $\begin{array}{l}\text { Nyssa javanica (Blume) } \\
\text { Wangerin }\end{array}$ & & Tree & Cornaceae & Fruit & Ripe fruits are eaten fresh & $\begin{array}{l}\text { Shuiyan } \\
\text { Village, } \\
\text { Ma'andi } \\
\text { Township }\end{array}$ & $\begin{array}{l}201,511- \\
07\end{array}$ & 2.3 & 0.44 \\
\hline $\begin{array}{l}\text { Swida macrophylla (Wall.) } \\
\text { Soják }\end{array}$ & & Tree & Cornaceae & Fruit & Used for pressing oil & $\begin{array}{l}\text { Xiaoxinjie } \\
\text { Township }\end{array}$ & LB-06 & 2.0 & 0.51 \\
\hline $\begin{array}{l}\text { Pouteria grandifolia (Wall.) } \\
\text { Baehni }\end{array}$ & & Tree & Sapotaceae & Fruit & Ripe fruits are eaten fresh & $\begin{array}{l}\text { Xiaoxinjie } \\
\text { Township }\end{array}$ & LB-02 & 1.9 & 0.52 \\
\hline $\begin{array}{l}\text { Diospyros lotus L. var. } \\
\text { mollissima C.Y. Wu }\end{array}$ & & Tree & Ebenaceae & Fruit & $\begin{array}{l}\text { Fruits eaten fresh, making } \\
\text { liquor or vinegar }\end{array}$ & $\begin{array}{l}\text { Lonajia } \\
\text { Village, Jiayin } \\
\text { Township }\end{array}$ & $\begin{array}{l}201,506- \\
36\end{array}$ & 2.4 & 0.58 \\
\hline Embelia ribes Burm.f. & & Shrub & Primulaceae & Fruit, & Fruit: eaten fresh; shoot: & Shuiyan & $201,511-$ & 2.3 & 0.42 \\
\hline
\end{tabular}


Table 3 Inventory of WEP species in Honghe terraced rice paddy system (Continued)

\begin{tabular}{|c|c|c|c|c|c|c|c|c|c|}
\hline Scientific name & $\begin{array}{l}\text { Vernacular } \\
\text { Name }\end{array}$ & $\begin{array}{l}\text { Life } \\
\text { form }\end{array}$ & Family name & $\begin{array}{l}\text { Parts } \\
\text { used }\end{array}$ & Preparation and uses & Study sites & $\begin{array}{l}\text { Voucher } \\
\text { number }\end{array}$ & $\mathrm{FUI}$ & UV \\
\hline & & & & shoot & potherb & $\begin{array}{l}\text { Village, } \\
\text { Ma'andi } \\
\text { Township }\end{array}$ & 14 & & \\
\hline $\begin{array}{l}\text { Embelia subcoriacea (C. B. } \\
\text { Clarke) Mez }\end{array}$ & & Shrub & Primulaceae & Fruit & Ripe fruits are eaten fresh & $\begin{array}{l}\text { Niujiaozhai } \\
\text { Township }\end{array}$ & $\begin{array}{l}201,606- \\
03\end{array}$ & 2.1 & 0.42 \\
\hline Maesa montana A. DC. & Ke tu a bo & Shrub & Primulaceae & Leaf & Tea substitute & $\begin{array}{l}\text { Lüchun } \\
\text { County }\end{array}$ & LB-20 & 0.2 & 0.39 \\
\hline Maesa parvifolia A. DC. & & Shrub & Primulaceae & Leaf & Tea substitute & $\begin{array}{l}\text { Lüchun } \\
\text { County }\end{array}$ & LB-19 & 1.4 & 0.37 \\
\hline Camellia pitardii Coh.-St. & & Shrub & Theaceae & Petal & Potherb & $\begin{array}{l}\text { Lüshuge } \\
\text { Village, Jiayin } \\
\text { Township }\end{array}$ & $\begin{array}{l}201,610- \\
21\end{array}$ & 2.7 & 0.40 \\
\hline $\begin{array}{l}\text { Actinidia kolomikta (Rupr. } \\
\text { and Maxim.) Maxim. }\end{array}$ & A zi ku nu & Shrub & Actinidiaceae & Fruit & Ripe fruits are eaten fresh & $\begin{array}{l}\text { Lonajia } \\
\text { Village, Jiayin } \\
\text { Township }\end{array}$ & $\begin{array}{l}201,506- \\
32\end{array}$ & 2.5 & 0.54 \\
\hline Saurauia napaulensis DC. & & Tree & Actinidiaceae & Fruit & Ripe fruits are eaten fresh & $\begin{array}{l}\text { Baohua } \\
\text { Township }\end{array}$ & $\begin{array}{l}201,511- \\
32\end{array}$ & 2.7 & 0.58 \\
\hline $\begin{array}{l}\text { Saurauia napaulensis DC. var. } \\
\text { montana C. F. Liang and Y. S. } \\
\text { Wang }\end{array}$ & & Tree & Actinidiaceae & Fruit & Ripe fruits are eaten fresh & $\begin{array}{l}\text { Lüshuge } \\
\text { Village, Jiayin } \\
\text { Township }\end{array}$ & $\begin{array}{l}201,610- \\
01\end{array}$ & 2.8 & 0.39 \\
\hline $\begin{array}{l}\text { Saurauia tristyla var. } \\
\text { hekouensis C. F. Liang and Y. } \\
\text { S. Wang }\end{array}$ & A nuo xi & Tree & Actinidiaceae & Fruit & Ripe fruits are eaten fresh & $\begin{array}{l}\text { Xiaoxinjie } \\
\text { Township }\end{array}$ & LB-05 & 2.7 & 0.42 \\
\hline $\begin{array}{l}\text { Gaultheria leucocarpa Bl. var. } \\
\text { crenulata (Kurz) T.Z.Hsu }\end{array}$ & Xie & Shrub & Ericaceae & Leaf & $\begin{array}{l}\text { Potherb (made into } \\
\text { soup) }\end{array}$ & $\begin{array}{l}\text { Xiaoxinjie } \\
\text { Township }\end{array}$ & LB-09 & 2.1 & 0.48 \\
\hline $\begin{array}{l}\text { Gaultheria longibracteolata } \\
\text { R.C.Fang }\end{array}$ & Ye lan mei & Shrub & Ericaceae & Fruit & Ripe fruits are eaten fresh & $\begin{array}{l}\text { Shuiyan } \\
\text { Village, } \\
\text { Ma'andi } \\
\text { Township }\end{array}$ & $\begin{array}{l}201,511- \\
09\end{array}$ & 1.9 & 0.45 \\
\hline Vaccinium bracteatum Thunb. & Ha na & Shrub & Ericaceae & Fruit & Ripe fruits are eaten fresh & $\begin{array}{l}\text { The junction } \\
\text { of Lüchun } \\
\text { County and } \\
\text { Yuanyang } \\
\text { County }\end{array}$ & $\begin{array}{l}201,506- \\
10\end{array}$ & 2.5 & 0.55 \\
\hline Pittosporopsis kerrii Craib & $\begin{array}{l}\text { Ha piao } \\
\text { mei che }\end{array}$ & Shrub & Icacinaceae & $\begin{array}{l}\text { Fruit, } \\
\text { seed }\end{array}$ & $\begin{array}{l}\text { Fruit: eaten fresh; seed: } \\
\text { roasted and eaten } \\
\text { (kernel) }\end{array}$ & $\begin{array}{l}\text { Lagu Village, } \\
\text { Sanmeng } \\
\text { Township }\end{array}$ & HHD-40 & 2.3 & 0.55 \\
\hline Canthium horridum Blume & $\begin{array}{l}\text { Ha da da } \\
\text { nue }\end{array}$ & Shrub & Rubiaceae & Fruit & Ripe fruits are eaten fresh & $\begin{array}{l}\text { Shuiyan } \\
\text { Village, } \\
\text { Ma'andi } \\
\text { Township }\end{array}$ & $\begin{array}{l}201,511- \\
05\end{array}$ & 2.4 & 0.51 \\
\hline Hedyotis tenelliflora Blume & $\begin{array}{l}\text { Gu suo na } \\
\text { ci }\end{array}$ & Herb & Rubiaceae & $\begin{array}{l}\text { Whole } \\
\text { plant }\end{array}$ & $\begin{array}{l}\text { Potherb (made into } \\
\text { soup) }\end{array}$ & $\begin{array}{l}\text { Lagu Village, } \\
\text { Sanmeng } \\
\text { Township }\end{array}$ & HHD-42 & 2.0 & 0.55 \\
\hline $\begin{array}{l}\text { Amalocalyx yunnanensis } \\
\text { Tsiang }\end{array}$ & & Liana & Apocynaceae & Fruit & $\begin{array}{l}\text { Young fruit slices are } \\
\text { eaten fresh with the } \\
\text { source made by pepper } \\
\text { and salt }\end{array}$ & $\begin{array}{l}\text { Lagu Village, } \\
\text { Sanmeng } \\
\text { Township }\end{array}$ & HHD-32 & 2.0 & \\
\hline $\begin{array}{l}\text { Dregea volubilis (L.f.) Benth. ex } \\
\text { Hook.f. }\end{array}$ & Ku cai & Liana & Apocynaceae & $\begin{array}{l}\text { Flower, } \\
\text { tender } \\
\text { leaf }\end{array}$ & Potherb & $\begin{array}{l}\text { Xiaoxinjie } \\
\text { Township }\end{array}$ & LB-12 & 3.0 & 0.72 \\
\hline Melodinus henryi Craib & $\begin{array}{l}\text { Ke se pa } \\
\text { ha }\end{array}$ & Liana & Apocynaceae & Fruit & Ripe fruits are eaten fresh & $\begin{array}{l}\text { Lüshuge } \\
\text { Village, Jiayin } \\
\text { Township }\end{array}$ & $\begin{array}{l}201,610- \\
06\end{array}$ & 2.6 & 0.45 \\
\hline $\begin{array}{l}\text { Lithospermum erythrorhizon } \\
\text { Siebold and Zucc. }\end{array}$ & & Herb & Boraginaceae & $\begin{array}{l}\text { Tender } \\
\text { stem, } \\
\text { leaf }\end{array}$ & Potherb & $\begin{array}{l}\text { Baohua } \\
\text { Township }\end{array}$ & HHD-26 & 2.0 & 0.50 \\
\hline
\end{tabular}


Table 3 Inventory of WEP species in Honghe terraced rice paddy system (Continued)

\begin{tabular}{|c|c|c|c|c|c|c|c|c|c|}
\hline Scientific name & $\begin{array}{l}\text { Vernacular } \\
\text { Name }\end{array}$ & $\begin{array}{l}\text { Life } \\
\text { form }\end{array}$ & Family name & $\begin{array}{l}\text { Parts } \\
\text { used }\end{array}$ & Preparation and uses & Study sites & $\begin{array}{l}\text { Voucher } \\
\text { number }\end{array}$ & FUI & UV \\
\hline $\begin{array}{l}\text { Lycium yunnanense Kuang } \\
\text { and A.M.Lu }\end{array}$ & & Shrub & Solanaceae & $\begin{array}{l}\text { tender } \\
\text { stem }\end{array}$ & Potherb & $\begin{array}{l}\text { Lonajia } \\
\text { Village, Jiayin } \\
\text { Township }\end{array}$ & $\begin{array}{l}201,506- \\
47\end{array}$ & 2.0 & 0.46 \\
\hline Solanum nigrum L. & Wo lun & Herb & Solanaceae & $\begin{array}{l}\text { Tender } \\
\text { leaf, fruit }\end{array}$ & $\begin{array}{l}\text { Fruit: eaten fresh; tender } \\
\text { leaf: potherb }\end{array}$ & $\begin{array}{l}\text { Xiongjia } \\
\text { Village, } \\
\text { Adebo } \\
\text { Township }\end{array}$ & $\begin{array}{l}\text { HHD- } \\
014\end{array}$ & 2.3 & 0.42 \\
\hline Solanum torvum Sw. & $\begin{array}{l}\text { Si ma ma } \\
\text { ha }\end{array}$ & Shrub & Solanaceae & Root & Potherb (usually stewed) & $\begin{array}{l}\text { Xiongjia } \\
\text { Village, } \\
\text { Adebo } \\
\text { Township }\end{array}$ & $\begin{array}{l}\text { HHD- } \\
018\end{array}$ & 2.9 & 0.46 \\
\hline Ligustrum sinense Lour. & $\begin{array}{l}\text { Ci kong ba } \\
\text { deng a bo }\end{array}$ & Tree & Oleaceae & Fruit & Liquor brewing & $\begin{array}{l}\text { Lagu Village, } \\
\text { Sanmeng } \\
\text { Township }\end{array}$ & HHD-46 & 0.6 & 0.05 \\
\hline $\begin{array}{l}\text { Rhynchotechum obovatum } \\
\text { (Griff.) B.L. Burtt }\end{array}$ & & Shrub & Gesneriaceae & Fruit & Ripe fruits are eaten fresh & $\begin{array}{l}\text { Lüshuge } \\
\text { Village, Jiayin } \\
\text { Township }\end{array}$ & $\begin{array}{l}201,610- \\
03\end{array}$ & 1.7 & 0.39 \\
\hline Plantago asiatica L. & Ka pae ca & Herb & Plantaginaceae & $\begin{array}{l}\text { Whole } \\
\text { plant }\end{array}$ & Potherb & $\begin{array}{l}\text { Xiongjia } \\
\text { Village, } \\
\text { Adebo } \\
\text { Township }\end{array}$ & $\begin{array}{l}201,506- \\
01\end{array}$ & 2.3 & 0.51 \\
\hline $\begin{array}{l}\text { Plantago asiatica L. subsp. } \\
\text { erosa (Wall.) Z. Y. Li }\end{array}$ & Ka pae ca & Herb & Plantaginaceae & $\begin{array}{l}\text { Tender } \\
\text { leaf }\end{array}$ & $\begin{array}{l}\text { Potherb (usually stewed } \\
\text { or made into soup) }\end{array}$ & $\begin{array}{l}\text { Baohua } \\
\text { Township }\end{array}$ & HHD-22 & 2.8 & 0.53 \\
\hline Plantago depressa Willd. & $\begin{array}{l}\text { Ha pa yu } \\
\text { cai }\end{array}$ & Herb & Plantaginaceae & $\begin{array}{l}\text { Whole } \\
\text { plant }\end{array}$ & $\begin{array}{l}\text { Potherb (soaked in water } \\
\text { and sir-fried) }\end{array}$ & $\begin{array}{l}\text { Xiaoxinjie } \\
\text { Township }\end{array}$ & LB-08 & 2.3 & 0.55 \\
\hline $\begin{array}{l}\text { Mayodendron igneum (Kurz) } \\
\text { Kurz }\end{array}$ & $\begin{array}{l}\text { A ci ma ha } \\
\text { nen }\end{array}$ & Tree & Bignoniaceae & Flower & Potherb & $\begin{array}{l}\text { Lüchun } \\
\text { County }\end{array}$ & LB-23 & 2.8 & 0.43 \\
\hline $\begin{array}{l}\text { Clinopodium chinense (Benth.) } \\
\text { Kuntze }\end{array}$ & $\begin{array}{l}\text { Zhaun } \\
\text { zhuan cai }\end{array}$ & Herb & Lamiaceae & $\begin{array}{l}\text { Tender } \\
\text { leaf, } \\
\text { tender } \\
\text { stem }\end{array}$ & Potherb & $\begin{array}{l}\text { Lagu Village, } \\
\text { Sanmeng } \\
\text { Township }\end{array}$ & HHD-50 & 2.4 & 0.44 \\
\hline Mentha canadensis $\mathrm{L}$. & $\begin{array}{l}\text { Wo zhi zhi } \\
\text { ma }\end{array}$ & Herb & Lamiaceae & $\begin{array}{l}\text { Tender } \\
\text { leaf, } \\
\text { tender } \\
\text { stem }\end{array}$ & Flavoring agent & $\begin{array}{l}\text { Shuiyan } \\
\text { Village, } \\
\text { Ma'andi } \\
\text { Township }\end{array}$ & $\begin{array}{l}201,511- \\
11\end{array}$ & 4.6 & 0.75 \\
\hline Rabdosia coetsoides C.Y.Wu & Nu ha ma & Herb & Lamiaceae & $\begin{array}{l}\text { Whole } \\
\text { plant }\end{array}$ & $\begin{array}{l}\text { Tea substitute or cooked } \\
\text { with meat (Potherb) }\end{array}$ & $\begin{array}{l}\text { Shuiyan } \\
\text { Village, } \\
\text { Ma'andi } \\
\text { Township }\end{array}$ & $\begin{array}{l}201,511- \\
15\end{array}$ & 0.7 & 0.23 \\
\hline $\begin{array}{l}\text { Helwingia japonica (Thunb.) } \\
\text { F.Dietr. }\end{array}$ & $\begin{array}{l}\text { Huo tie tie } \\
\text { du }\end{array}$ & Shrub & Helwingiaceae & $\begin{array}{l}\text { Tender } \\
\text { stem, } \\
\text { leaf }\end{array}$ & $\begin{array}{l}\text { Potherb (blanched in hot } \\
\text { water, then soaked in } \\
\text { cold water before } \\
\text { cooking) }\end{array}$ & $\begin{array}{l}\text { Niujiaozhai } \\
\text { Township }\end{array}$ & $\begin{array}{l}201,606- \\
18\end{array}$ & 1.9 & 0.53 \\
\hline $\begin{array}{l}\text { Campanumoea javanica } \\
\text { Blume }\end{array}$ & $\begin{array}{l}\text { A mi nan } \\
\text { guo }\end{array}$ & Liana & Campanulaceae & Fruit & Ripe fruits are eaten fresh & $\begin{array}{l}\text { Lagu Village, } \\
\text { Sanmeng } \\
\text { Township }\end{array}$ & HHD-36 & 1.6 & 0.56 \\
\hline Lobelia angulata G.Forst. & & Herb & Campanulaceae & $\begin{array}{l}\text { Tender } \\
\text { leaf, } \\
\text { tender } \\
\text { stem }\end{array}$ & Potherb & $\begin{array}{l}\text { Shuiyan } \\
\text { Village, } \\
\text { Ma'andi } \\
\text { Township }\end{array}$ & $\begin{array}{l}201,511- \\
31\end{array}$ & 1.8 & 0.42 \\
\hline $\begin{array}{l}\text { Adenocaulon himalaicum } \\
\text { Edgew. }\end{array}$ & $\begin{array}{l}\text { Bu lü wu } \\
\text { hu }\end{array}$ & Herb & Asteraceae & $\begin{array}{l}\text { Tender } \\
\text { leaf }\end{array}$ & Potherb & $\begin{array}{l}\text { Niujiaozhai } \\
\text { Township }\end{array}$ & $\begin{array}{l}201,606- \\
15\end{array}$ & 1.8 & 0.45 \\
\hline Bidens pilosa $\mathrm{L}$. & $\begin{array}{l}\text { Hei ni zuo } \\
\text { ge mo }\end{array}$ & Herb & Asteraceae & $\begin{array}{l}\text { Tender } \\
\text { leaf }\end{array}$ & $\begin{array}{l}\text { Potherb (stewed until it } \\
\text { is tender) }\end{array}$ & $\begin{array}{l}\text { Qingkou } \\
\text { Village, Xinjie } \\
\text { Township }\end{array}$ & $\begin{array}{l}201,506- \\
26\end{array}$ & 2.0 & 0.53 \\
\hline $\begin{array}{l}\text { Cirsium japonicum (Thunb.) } \\
\text { Fisch. ex DC. }\end{array}$ & $\begin{array}{l}\text { Che pei a } \\
\text { gong }\end{array}$ & Herb & Asteraceae & Root & $\begin{array}{l}\text { Stewed with pork for } \\
\text { nourishing }\end{array}$ & $\begin{array}{l}\text { Baohua } \\
\text { Township }\end{array}$ & HHD-21 & 2.9 & 0.43 \\
\hline
\end{tabular}


Table 3 Inventory of WEP species in Honghe terraced rice paddy system (Continued)

\begin{tabular}{|c|c|c|c|c|c|c|c|c|c|}
\hline Scientific name & $\begin{array}{l}\text { Vernacular } \\
\text { Name }\end{array}$ & $\begin{array}{l}\text { Life } \\
\text { form }\end{array}$ & Family name & $\begin{array}{l}\text { Parts } \\
\text { used }\end{array}$ & Preparation and uses & Study sites & $\begin{array}{l}\text { Voucher } \\
\text { number }\end{array}$ & FUI & UV \\
\hline $\begin{array}{l}\text { Crassocephalum crepidioides } \\
\text { (Benth.) S. Moore }\end{array}$ & O mi o sa & Herb & Asteraceae & $\begin{array}{l}\text { Tender } \\
\text { leaf }\end{array}$ & Potherb & $\begin{array}{l}\text { Baohua } \\
\text { Township }\end{array}$ & HHD-29 & 3.9 & 0.76 \\
\hline Eclipta prostrata (L.) L. & $\begin{array}{l}\text { A ji mei, a } \\
\text { ge wo chi }\end{array}$ & Herb & Asteraceae & $\begin{array}{l}\text { Tender } \\
\text { leaf }\end{array}$ & Potherb & $\begin{array}{l}\text { Qingkou } \\
\text { Village, Xinjie } \\
\text { Township }\end{array}$ & $\begin{array}{l}201,506- \\
25\end{array}$ & 2.2 & 0.49 \\
\hline Gnaphalium affine D. Don & $\begin{array}{l}\text { A mi sha } \\
\text { chu }\end{array}$ & Herb & Asteraceae & $\begin{array}{l}\text { Leaf, } \\
\text { stem }\end{array}$ & Potherb & $\begin{array}{l}\text { Qingkou } \\
\text { Village, Xinjie } \\
\text { Township }\end{array}$ & $\begin{array}{l}201,506- \\
23\end{array}$ & 2.1 & 0.42 \\
\hline Ixeris polycephala Cass. & & Herb & Asteraceae & $\begin{array}{l}\text { Tender } \\
\text { leaf, } \\
\text { tender } \\
\text { stem }\end{array}$ & $\begin{array}{l}\text { Potherb (blanched before } \\
\text { eating and making salad) }\end{array}$ & $\begin{array}{l}\text { Lüchun } \\
\text { County }\end{array}$ & LB-26 & 3.9 & 0.75 \\
\hline Lactuca serriola L. & & Herb & Asteraceae & $\begin{array}{l}\text { Tender } \\
\text { leaf, } \\
\text { tender } \\
\text { stem }\end{array}$ & Potherb & $\begin{array}{l}\text { Xiaoxinjie } \\
\text { Township }\end{array}$ & LB-13 & 2.8 & 0.52 \\
\hline Lagedium sibiricum (L.) Soják & $\begin{array}{l}\text { E si lao } \\
\text { gong zi }\end{array}$ & Herb & Asteraceae & $\begin{array}{l}\text { Tender } \\
\text { leaf, } \\
\text { tender } \\
\text { stem }\end{array}$ & Potherb & $\begin{array}{l}\text { Shuiyan } \\
\text { Village, } \\
\text { Ma'andi } \\
\text { Township }\end{array}$ & $\begin{array}{l}201,511- \\
29\end{array}$ & 2.8 & 0.54 \\
\hline $\begin{array}{l}\text { Laggera pterodonta (DC.) } \\
\text { Sch.Bip. ex Oliv. }\end{array}$ & $\begin{array}{l}\text { Wo sa la } \\
\text { ma }\end{array}$ & Herb & Asteraceae & $\begin{array}{l}\text { Whole } \\
\text { plant }\end{array}$ & $\begin{array}{l}\text { Potherb (blanched in hot } \\
\text { water, then soaked in } \\
\text { cold water before } \\
\text { cooking) }\end{array}$ & $\begin{array}{l}\text { Lüchun } \\
\text { County }\end{array}$ & LB-25 & 2.9 & 0.51 \\
\hline Viburnum dilatatum Thunb. & $\begin{array}{l}\text { Pu tong a } \\
\text { bo }\end{array}$ & Shrub & Adoxaceae & Fruit & $\begin{array}{l}\text { Fruits eaten fresh or } \\
\text { liquor brewing }\end{array}$ & $\begin{array}{l}\text { Qingkou } \\
\text { Village, Xinjie } \\
\text { Township }\end{array}$ & $\begin{array}{l}201,506- \\
16\end{array}$ & 2.7 & 0.51 \\
\hline $\begin{array}{l}\text { Dipsacus asperoides } \\
\text { C.Y.Cheng and T.M.Ai }\end{array}$ & $\begin{array}{l}\text { Pao tou } \\
\text { cao }\end{array}$ & Herb & Caprifoliaceae & $\begin{array}{l}\text { Tender } \\
\text { leaf, root }\end{array}$ & $\begin{array}{l}\text { Potherb (Usually stewed } \\
\text { with pork or made into } \\
\text { soup) }\end{array}$ & $\begin{array}{l}\text { Baohua } \\
\text { Township }\end{array}$ & HHD-23 & 2.7 & 0.51 \\
\hline Valeriana jatamansi Jones & $\begin{array}{l}\text { Ye zuo } \\
\text { zuo pu }\end{array}$ & Herb & Caprifoliaceae & $\begin{array}{l}\text { Flower, } \\
\text { root }\end{array}$ & $\begin{array}{l}\text { Flower: eaten fresh } \\
\text { (Potherb); root: stewed } \\
\text { for nourishing }\end{array}$ & $\begin{array}{l}\text { Lonajia } \\
\text { Village, Jiayin } \\
\text { Township }\end{array}$ & $\begin{array}{l}201,506- \\
40\end{array}$ & 1.7 & 0.51 \\
\hline $\begin{array}{l}\text { Acanthopanax trifoliatus (L.) } \\
\text { Voss }\end{array}$ & Jiu duo & Shrub & Araliaceae & $\begin{array}{l}\text { Tender } \\
\text { stem }\end{array}$ & Potherb & $\begin{array}{l}\text { Lüchun } \\
\text { County }\end{array}$ & LB-24 & 2.5 & 0.50 \\
\hline $\begin{array}{l}\text { Eleutherococcus senticosus } \\
\text { (Rupr. et Maxim.) Maxim. }\end{array}$ & & Shrub & Araliaceae & $\begin{array}{l}\text { Tender } \\
\text { stem, } \\
\text { leaf }\end{array}$ & Potherb & $\begin{array}{l}\text { Lüshuge } \\
\text { Village, Jiayin } \\
\text { Township }\end{array}$ & $\begin{array}{l}201,610- \\
18\end{array}$ & 2.3 & 0.42 \\
\hline Centella asiatica (L.) Urb. & $\begin{array}{l}\text { Ban chao } \\
\text { wo ba }\end{array}$ & Herb & Apiaceae & $\begin{array}{l}\text { Tender } \\
\text { leaf, } \\
\text { tender } \\
\text { stem }\end{array}$ & Potherb & $\begin{array}{l}\text { Shuiyan } \\
\text { Village, } \\
\text { Ma'andi } \\
\text { Township }\end{array}$ & $\begin{array}{l}201,511- \\
27\end{array}$ & 3.0 & 0.58 \\
\hline Eryngium foetidum L. & Ga la ya so & Herb & Apiaceae & $\begin{array}{l}\text { Tender } \\
\text { leaf, } \\
\text { tender } \\
\text { stem }\end{array}$ & $\begin{array}{l}\text { Flavoring agent or stir- } \\
\text { fried (potherb) }\end{array}$ & $\begin{array}{l}\text { Qingkou } \\
\text { Village, Xinjie } \\
\text { Township }\end{array}$ & $\begin{array}{l}201,506- \\
18\end{array}$ & 1.9 & 0.52 \\
\hline $\begin{array}{l}\text { Ligusticum chuanxiong } \\
\text { S.H.Qiu, Y.Q.Zeng, K.Y.Pan, } \\
\text { Y.C.Tang, and J.M.Xu }\end{array}$ & $\begin{array}{l}\text { Tong e } \\
\text { jian sa }\end{array}$ & Herb & Apiaceae & $\begin{array}{l}\text { Tender } \\
\text { leaf }\end{array}$ & $\begin{array}{l}\text { Flavoring agent or stir- } \\
\text { fried (potherb) }\end{array}$ & $\begin{array}{l}\text { Qingkou } \\
\text { Village, Xinjie } \\
\text { Township }\end{array}$ & $\begin{array}{l}201,506- \\
17\end{array}$ & 2.6 & 0.53 \\
\hline $\begin{array}{l}\text { Oenanthe javanica (Blume) } \\
\text { DC. }\end{array}$ & Zha suo & Herb & Apiaceae & $\begin{array}{l}\text { Tender } \\
\text { leaf, } \\
\text { tender } \\
\text { stem }\end{array}$ & Potherb & $\begin{array}{l}\text { Niujiaozhai } \\
\text { Township }\end{array}$ & $\begin{array}{l}201,606- \\
14\end{array}$ & 4.3 & 0.82 \\
\hline $\begin{array}{l}\text { Sanicula astrantiifolia H. Wolff } \\
\text { ex Kretschmer }\end{array}$ & $\begin{array}{l}\text { Xiao hei } \\
\text { yao }\end{array}$ & Herb & Apiaceae & $\begin{array}{l}\text { Whole } \\
\text { plant }\end{array}$ & Potherb (usually sir-fried) & $\begin{array}{l}\text { Lonajia } \\
\text { Village, Jiayin } \\
\text { Township }\end{array}$ & $\begin{array}{l}201,506- \\
54\end{array}$ & 2.9 & 0.48 \\
\hline
\end{tabular}


Table 3 Inventory of WEP species in Honghe terraced rice paddy system (Continued)

\begin{tabular}{|c|c|c|c|c|c|c|c|c|c|}
\hline Scientific name & $\begin{array}{l}\text { Vernacular } \\
\text { Name }\end{array}$ & $\begin{array}{l}\text { Life } \\
\text { form }\end{array}$ & Family name & $\begin{array}{l}\text { Parts } \\
\text { used }\end{array}$ & Preparation and uses & Study sites & $\begin{array}{l}\text { Voucher } \\
\text { number }\end{array}$ & FUI & UV \\
\hline Lygodium digitatum C. Presl & $\begin{array}{l}\text { Ha da da } \\
\text { xiu }\end{array}$ & Liana & Lygodiaceae & $\begin{array}{l}\text { Tender } \\
\text { stem }\end{array}$ & Potherb & $\begin{array}{l}\text { Lüshuge } \\
\text { Village, Jiayin } \\
\text { Township }\end{array}$ & $\begin{array}{l}201,610- \\
17\end{array}$ & 2.5 & 0.54 \\
\hline $\begin{array}{l}\text { Pteridium aquilinum var. } \\
\text { latiusculum (Desv.) Underw. } \\
\text { ex A. Heller }\end{array}$ & Ye qie & Herb & Dennstaedtiaceae & Shoot & Potherb & $\begin{array}{l}\text { Shuiyan } \\
\text { Village, } \\
\text { Ma'andi } \\
\text { Township }\end{array}$ & $\begin{array}{l}201,511- \\
26\end{array}$ & 2.7 & 0.45 \\
\hline $\begin{array}{l}\text { Ceratopteris thalictroides (L.) } \\
\text { Brongn. }\end{array}$ & Da lie & Herb & Pteridiaceae & Shoot & Potherb & $\begin{array}{l}\text { Niujiaozhai } \\
\text { Township }\end{array}$ & $\begin{array}{l}201,606- \\
17\end{array}$ & 2.6 & 0.47 \\
\hline $\begin{array}{l}\text { Callipteris esculenta (Retz.) J. } \\
\text { Sm. ex T. Moore and } \\
\text { Houlston }\end{array}$ & & Herb & Athyriaceae & $\begin{array}{l}\text { Tender } \\
\text { leaf }\end{array}$ & Potherb & $\begin{array}{l}\text { Lagu Village, } \\
\text { Sanmeng } \\
\text { Township }\end{array}$ & HHD-47 & 2.5 & 0.56 \\
\hline $\begin{array}{l}\text { Callipteris esculenta var. } \\
\text { pubescens (Link) Ching }\end{array}$ & & Herb & Athyriaceae & $\begin{array}{l}\text { Tender } \\
\text { leaf }\end{array}$ & Potherb & $\begin{array}{l}\text { Baohua } \\
\text { Township }\end{array}$ & HHD-24 & 1.8 & 0.56 \\
\hline $\begin{array}{l}\text { Gymnocarpium } \\
\text { remotepinnatum (Hayata) } \\
\text { Ching }\end{array}$ & $\mathrm{Ha}$ & Herb & Athyriaceae & $\begin{array}{l}\text { Tender } \\
\text { leaf }\end{array}$ & Potherb & $\begin{array}{l}\text { The junction } \\
\text { of Lüchun } \\
\text { County and } \\
\text { Yuanyang } \\
\text { County }\end{array}$ & $\begin{array}{l}201,506- \\
12\end{array}$ & 2.1 & 0.58 \\
\hline $\begin{array}{l}\text { Parathelypteris glanduligera } \\
\text { (Kunze) Ching }\end{array}$ & Ha da & Herb & Thelypteridaceae & Shoot & Potherb & $\begin{array}{l}\text { Lonajia } \\
\text { Village, Jiayin } \\
\text { Township }\end{array}$ & $\begin{array}{l}201,506- \\
49\end{array}$ & 2.7 & 0.56 \\
\hline Marsilea quadrifolia L. & $\begin{array}{l}\text { He dou a } \\
\text { ya mo }\end{array}$ & Herb & Marsileaceae & $\begin{array}{l}\text { Tender } \\
\text { leaf }\end{array}$ & Potherb & $\begin{array}{l}\text { Shuiyan } \\
\text { Village, } \\
\text { Ma'andi } \\
\text { Township }\end{array}$ & $\begin{array}{l}201,511- \\
25\end{array}$ & 3.3 & 0.59 \\
\hline
\end{tabular}

The order of plant species in this table is followed by the APG IV system, gymnosperms classification system (1978), and Qinrenchang fern plant classification system (1978)

local people scrape off the exudate from its tuber and apply this exudate directly onto wounds for wound healing $[36,37]$. The exudate has high polysaccharide content and possesses good antioxidant bioactivity [36, 37].

The UV and FUI value of WEP species in the Honghe area Quantitative analyses were calculated to determine the local importance of each wild edible species. The use values (UV) and frequency of utilization indices (FUI) of each species were calculated. The 20 WEP species with the highest UV are listed along with their average FUI in Table 7.

Litsea pungens had the highest UV and average FUI (Table 7). Because of its unique flavor and positive effects on human health, it has become the most commonly used edible species as a spice. Some local people even use the

Table 4 Life forms of WEP species in Honghe terraced rice paddy system

\begin{tabular}{lll}
\hline Life form & Records & Percentage \\
\hline Herbs & 79 & $35.3 \%$ \\
Trees & 68 & $30.4 \%$ \\
Shrubs & 46 & $20.5 \%$ \\
Lianas & 21 & $9.4 \%$ \\
Bamboo & 10 & $4.5 \%$ \\
\hline
\end{tabular}

oil extracted from this species to repel mosquitos. Based on both local medical theory and scientific research, $L$. pungens can help to promote appetite and improve digestion [38]. Mentha canadensis is also a popular spice among local people, especially for cooking meat. However, its average FUI value and UV are relatively lower than for L. pungens. The second highest UV belongs to Phyllanthus emblica, while its average FUI value is similar to that of $L$. pungens. The high UV and average FUI values of

Table 5 Edible parts of WEP species in Honghe terraced rice paddy system

\begin{tabular}{lll}
\hline Part used & Records & Percentage \\
\hline Fruit & 98 & $43.8 \%$ \\
Stem and leaf & 83 & $37.1 \%$ \\
Shoot & 18 & $8.0 \%$ \\
Seed & 18 & $8.0 \%$ \\
Flower (petal, bract) & 16 & $7.1 \%$ \\
Whole plant & 8 & $3.6 \%$ \\
Root & 6 & $2.7 \%$ \\
Rhizome & 3 & $1.3 \%$ \\
Tuber & 3 & $1.3 \%$ \\
Bark & 1 & $0.4 \%$ \\
\hline
\end{tabular}


Table 6 Preparation and uses of WEP species in Honghe terraced rice paddy system

\begin{tabular}{lll}
\hline Preparation and uses & Records & Percentage \\
\hline Potherb & 95 & $42.4 \%$ \\
Eaten fresh & 84 & $37.5 \%$ \\
Flavoring agent & 16 & $7.1 \%$ \\
Nuts & 12 & $5.4 \%$ \\
Tea substitute & 11 & $4.9 \%$ \\
Bamboo shoots & 10 & $4.5 \%$ \\
Liquor brewing & 8 & $3.6 \%$ \\
Grain substitute & 2 & - \\
Special tofu & 2 & - \\
Sweetener & 1 & - \\
\hline
\end{tabular}

P. emblica may be attributed to its juicy and tasty fruits and its special cultural property: its tender bark is consumed in every October Festival and God Walled Festival.

Several other plants were found to be popular as wild vegetables in our study area based on their high UV and average FUI, including the following: Acacia pennata, Ixeris polycephala, Amaranthus viridis, Centella asiatica, Colocasia gigantea, Crassocephalum crepidioides, Dregea volubilis, Elatostema involucratum, Houttuynia cordata, Oenanthe javanica, and Zingiber striolatum. For example, $H$. cordata is the most common wild vegetable in Southern China. Many modern scientific studies have indicated that this species possesses excellent antimicrobial, anti-cancer, anti-inflammatory, and immunoenhancement bioactivities $[39,40]$. C. gigantea is also a popular food plant, especially in Southwest China, and we found that the stem of $C$. gigantean contains high levels of nutrients and no heavy metals [41]. In addition, the inflorescence of Musa acuminata and Zingiber striolatum and the fruits of Ficus auriculata, Baccaurea ramiflora, and Musa acuminata are all relatively popular and important in local daily life.

\section{The effect of WEP species on communities' economic income}

Based on our interviews with key informants in local markets, the trading volume of WEP species was on a very small scale (less than 15 yuan each stall), which means selling WEP species could only bring a small income supplement and was usually unstable for local people. Therefore, driven by economic profits, many villagers have switched from growing rice to other economic plants like bananas in Hani terraced rice paddy fields. Many Hani informants reported that planting bananas can bring more income than growing rice. While in the short term, Hani farmers can get a higher income by growing bananas; they have to dry the rice paddy fields before planting bananas,

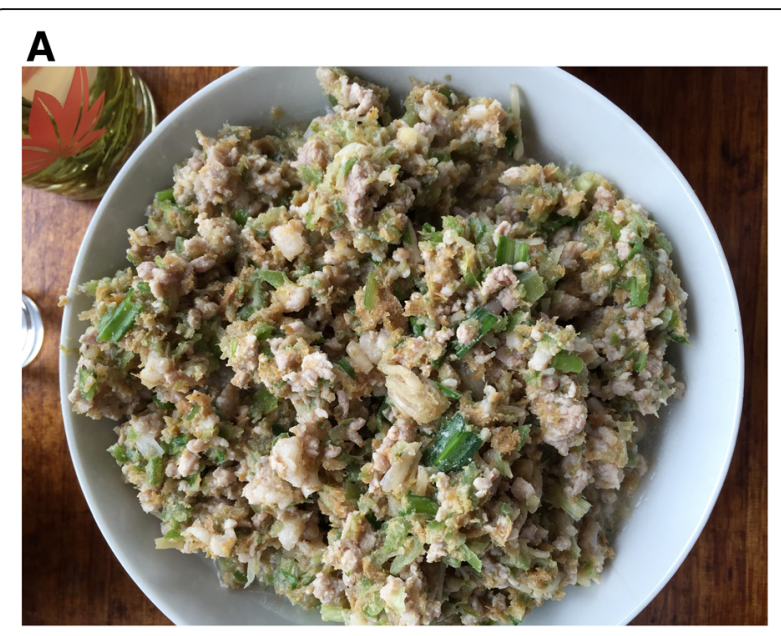

B
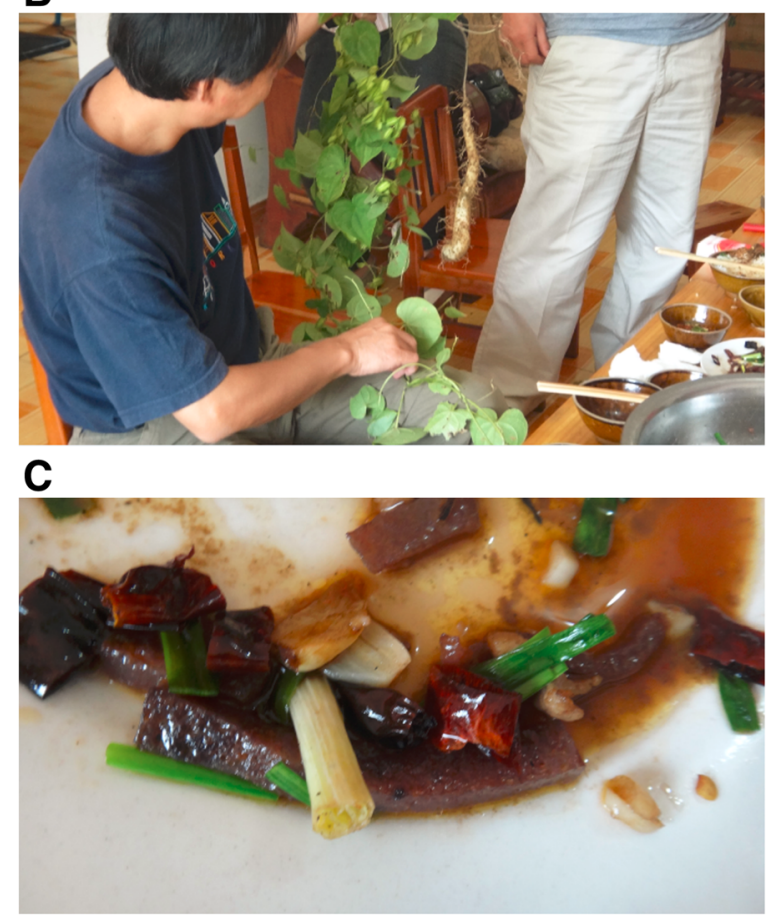

Fig. 4 Dish of Phyllanthus emblica (a). Dioscorea subcalva and dish of D. subcalva $(\mathbf{b}, \mathbf{c})$

which is against the Hani traditional ideas of sustainability. In doing so, the original construction and wetland habitats, as well as biodiversity, will be destroyed in the long term. Nevertheless, according to our observations, drying rice paddy to plant others was shown in different places in the Honghe region, especially in Jinping County.

The sources of and threats to the diversity of WEP species in the Honghe region

Our investigation revealed the diversity of WEP species in the Honghe area. Several reasons contributing to local biodiversity and WEP diversity have been analyzed 
Table 7 Top 20 WEP species with highest use value in Honghe terraced rice paddy system

\begin{tabular}{|c|c|c|c|}
\hline Scientific name & Preparation and uses & FUI & UV \\
\hline Litsea pungens & Flavoring agent & 4.9 & 0.92 \\
\hline Phyllanthus emblica & $\begin{array}{l}\text { Fruit: eaten fresh; bark: special } \\
\text { dishes }\end{array}$ & 4.9 & 0.90 \\
\hline Baccaurea ramiflora & Ripe fruits are eaten fresh & 4.2 & 0.89 \\
\hline Houttuynia cordata & Potherb or flavoring agent & 4.5 & 0.89 \\
\hline Oenanthe javanica & Potherb & 4.3 & 0.82 \\
\hline Colocasia gigantea & Potherb (cooked thoroughly) & 4.0 & 0.80 \\
\hline Crassocephalum crepidioides & Potherb & 3.9 & 0.76 \\
\hline Crateva unilocularis & Made into pickles (preserved) & 3.9 & 0.75 \\
\hline Mentha canadensis & Flavoring agent & 4.6 & 0.75 \\
\hline Ixeris polycephala & Potherb & 4.0 & 0.75 \\
\hline Zingiber striolatum & Potherb & 3.0 & 0.73 \\
\hline Dregea volubilis & Potherb & 3.0 & 0.72 \\
\hline Musa acuminata & $\begin{array}{l}\text { Fruit: eaten fresh; flower and pith } \\
\text { part: potherb }\end{array}$ & 3.8 & 0.70 \\
\hline Ficus auriculata & Ripe fruits are eaten fresh & 3.4 & 0.68 \\
\hline Nasturtium officinale & Potherb & 3.4 & 0.66 \\
\hline Acacia pennata & Potherb & 3.4 & 0.65 \\
\hline Elatostema involucratum & Potherb & 3.3 & 0.65 \\
\hline Amaranthus viridis & Potherb & 3.6 & 0.61 \\
\hline Marsilea quadrifolia & Potherb & 3.3 & 0.59 \\
\hline Centella asiatica & Potherb & 3.0 & 0.58 \\
\hline
\end{tabular}

(Fig. 5). The varied natural geographic environments and weather conditions are two of the main reasons for the diversity of WEP species. Secondly, the sustainable landscape structure constructed by the locals, including the four critical elements of forests, villages, terraces, and rivers, has a robust regulating ability, particularly for the regulation of essential water resources. Additionally, with the guidance of abundant traditional knowledge concerning reasonable agricultural management, excellent ecological benefits have been made to improve and maintain stability and biodiversity in the whole agroecosystem. Some Hani taboos, village regulations, and non-

\section{Positive Contributions}

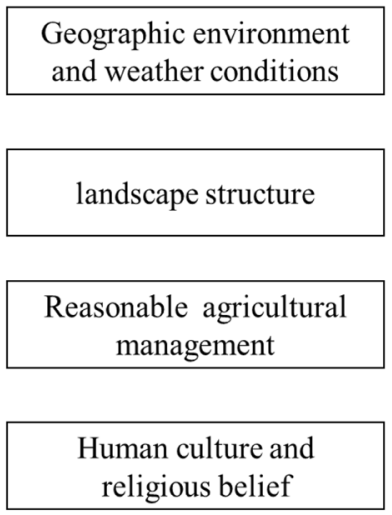

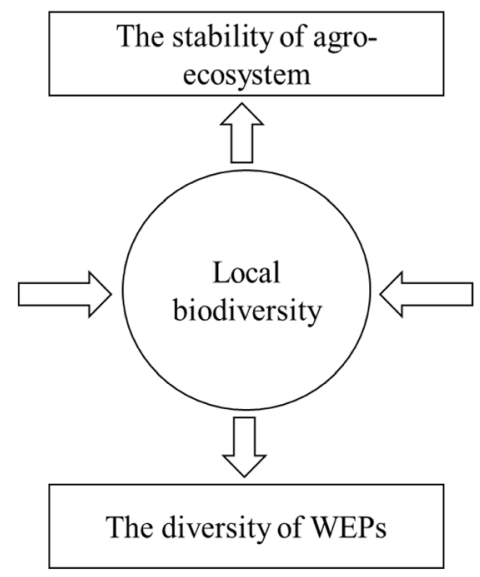

\section{Negative Contributions}

Lack of young labours

The use of pesticides and chemical fertilizers

excessive exploitation of natural resources

changing terraces fields into economic fields

Fig. 5 The relationship between local biodiversity and its effect factors 
governmental agreements, as well as local religious beliefs like the worship of the mountain deity and magic woods, have restrained the behaviors of local people and protected the surroundings.

However, the stability of Hani terraced rice paddy fields, which has been maintained for centuries, is now facing a series of challenges (Fig. 5). In our research area, traditional rice planting methods have been damaged by modernization. Based on our investigation, local young people prefer to work in urban areas to make more money instead of doing farm works in their hometowns. Traditional knowledge related to farmland management is only mastered by the older generation and is fading away rapidly (Table 3), and $18 \%$ of recorded species lack local Hani names. One reason for this lack of local names is that the Hani people are traditionally illiterate so that traditional knowledge can only be passed on orally by generations, so this knowledge is vulnerable to loss via acculturation. Although a writing system of the Hani language has been in place since 1957, it has not been widely adopted in the Hani communities. In addition, large-scale growing of hybrid rice requires less use of traditional agricultural methods and instead relies on pesticides and chemical fertilizers. Furthermore, the excessive exploitation of natural resources and the drying and changing of traditional rice terraces fields into economic fields are becoming increasingly frequent in the Honghe area nowadays. These phenomena are leading to a sharp decrease in the diversity of traditional knowledge and local biodiversity, which might damage the agroecosystem in this region [42, 43].

Since 2013, when the Hani agroecosystem was elected into the UNESCO World Heritage List, tourism has increased markedly. In 2015, the tourism industry generated about 191.5 billion yuan and accounted for about $70 \%$ of local government revenues (out of 275.6 billion yuan). However, the local villagers have obtained minimal economic benefits from the local tourism industry. The traditional agroecosystem cannot sustain the daily food needs of the local people anymore. The Hani are now turning to the tourism industry, which may help to protect the traditional knowledge and biodiversity in this agroecosystem.

\section{Conclusion}

An ethnobotanical study on WEP species from the Hani terraced rice paddy agroecosystems in Southeast Yunnan, China, was conducted. Two hundred and twentyfive species (belonging to 170 genera and 90 families) of wild edible species and the information of their life forms, edible parts, and preparation methods were documented. Based on our analysis, the most widely eaten parts of WEP species are fruits, stem, and leaves. The most common processing methods for WEP species are cooking them as a potherb or eating them fresh. These results are closely related to the local lifestyle and reflect the local biodiversity. The use values (UV) of WEP species were also calculated, and the 20 species with the highest use value were listed. Compared with other WEP species, these 20 species are relatively more important to local daily life, and Litsea pungens, a local common natural spice, is the most popular WEP based on its high UV metric.

The reasons for local biodiversity and the challenges for local agroecosystem have been analyzed. This agroecosystem is facing severe problems concerning natural resource conservation, environmental protection, and the economic development of local communities in this agroecosystem. Prestigious designations like UNESCO World Heritage Site have helped to promote ecotourism, which has begun to improve the livelihood of local people while sustaining the operation of this agroecosystem.

In conclusion, there are abundant plant resources in the Hani terraced rice paddy field system because it is an ancient sustainable agroecosystem. However, in modern times, this region has suffered a series of threats. It is, therefore, critical to develop an effective way to protect it and to ensure its sustainability for its inhabitants.

\section{Acknowledgements \\ We thank Prof. Edward Kennelly from the City University of New York and Mr. Eric Miller from the University of California at San Fransisco, who edited the English language. We are very grateful to the local people in Honghe Hani terraced rice paddy agroecosystem, Yunnan Province, who provided valuable information about local wild edible plants.}

\section{Authors' contributions}

CLL conceived and designed the study. BSL, CLL, BL, HZZ, HKZ, XL, LJM, YZW, YJB, XBZ, JQL, and JY conducted data collection. CLL, BL, and JY identified wild edible plants. BSL interpreted and analyzed data and wrote the draft manuscript. All authors read and approved the final manuscript.

\section{Funding}

This work was financially supported by the National Natural Science Foundation of China (31761143001 and 31870316), Biodiversity Survey and Assessment Project of the Ministry of Ecology and Environment of China (2019HJ2096001006), Key Laboratory of Ethnomedicine (Minzu University of China) of Ministry of Education of China (KLEM-ZZ201906 and KLEMZZ201806), Minzu University of China (Collaborative Innovation Center for Ethnic Minority Development and yldxxk201819), Ministry of Education of China and State Administration of Foreign Experts Affairs of China (B08044), and Bureau of World Heritage of Honghe Prefecture.

Availability of data and materials

All data generated or analyzed during this study are included in this published article (and its supplementary information files).

Ethics approval and consent to participate Not applicable.

Consent for publication

Not applicable.

Competing interests

The authors declare that they have no competing interests. 


\section{Author details}

College of Life and Environmental Sciences, Minzu University of China, Beijing 100081, China. ${ }^{2}$ Key Laboratory of Ethnomedicine (Minzu University of China), Ministry of Education, Beijing 100081, China. ${ }^{3}$ Bureau of Word Heritage of Honghe Prefecture, Mengzi 661100, Yunnan, China. ${ }^{4}$ Kunming Institute of Botany, Chinese Academy of Sciences, Kunming 650201, China.

Received: 12 September 2019 Accepted: 21 October 2019

\section{Published online: 27 November 2019}

\section{References}

1. Min QW, Yuan Z, He L. Hani terraced fields: a agriculture, ecology and culture multiplexed system. China Cult Herit. 2013;3:12-8.

2. Hu WY, Shen Q. Evaluation on landscape stability of Yuanyang Hani terrace. Yunnan Geogr Environ Res. 2011;1:11-7. https://doi.org/10.3969/ j.issn.1001-7852.2011.01.003.

3. Yao M, Cui BS. The vertical characteristics of ecosystem of Hani's terrace paddyfield in Yunnan, China. Acta Ecol Sin. 2006;7:2115-24. https://doi.org/ 10.3321/j.issn:1000-0933.2006.07.009.

4. Ju Y, Zhuo JX, Liu B, Long CL. Eating from the wild: diversity of wild edible plants used by Tibetans in Shangri-la region, Yunnan, China. J Ethnobiol Ethnomed. 2012;1:28. https://doi.org/10.1186/1746-4269-9-28.

5. Ying BY, Wen MQ, Cheng LM, Zheng Y, Tao XY, Zhi C, Jing L. Resilience of the Hani rice terraces system to extreme drought. J Food Agric Environ. 2013;11:2376-82. https://doi.org/10.1234/4.2013.5051.

6. Zhu YY, Chen HR, Fan JH, Wang YY. Genetic diversity and disease control in rice. Nature. 2000;6797:718. https://doi.org/10.1038/35021046.

7. Li FF, Pan B, Zhang D, Yang XL, Li H, Liao SH, Ghaffar A, Peng HB, Xing BS. Organic matter source and degradation as revealed by molecular biomarkers in agricultural soils of Yuanyang terrace. Sci Rep. 2015;5:11074. https://doi.org/10.1038/srep11074.

8. Compilation Committee of Yearbook of Honghe Prefecture: Yearbook of Honghe Prefecture. Kunming, Yunnan, China: The Peoples Press of Yunnan; 2015

9. Jiao YM, Chen GD, Xiao DN. A study on the cultural landscape of Hani's terrace and its protection. Geogr Res. 2002;6:733-41. https://doi.org/10. 3321/j.issn:1000-0585.2002.06.009.

10. Shi JC. The Hani terraced field at Honghe: typical Chinese wetland. J Yunnan National Univ. 2004;5:18. https://doi.org/10.3969/j.issn.1672-867X.2004.05.018.

11. Feng JC, Shi S, He SJ. Hani terrace ecosystem in Yunnan province. J CUN (Nat Sci Edit). 2008;17:146-52.

12. Jiao YM, Chen GD, Xiao DN. Ascertainment of the stable mechanism of terrace landscape in subtropic mountain areas_— - a case study of Hani terrace landscape in Yuanyang county. J Yunnan Norm Univ. 2003;23:55-60.

13. Heywood VH. Ethnopharmacology, food production, nutrition and biodiversity conservation: towards a sustainable future for indigenous peoples. J Ethnopharmacol. 2011;1:1-15. https://doi.org/10.1016/j.jep.2011.05.027.

14. Hadjichambis AC, Paraskeva-Hadjichambi D, Della A, Elena Giusti M, De Pasquale C, Lenzarini C, Censorii E, Reyes Gonzales-Tejero M, Patricia Sanchez-Rojas C, Ramiro-Gutierrez JM, Skoula M. Wild and semi-domesticated food plant consumption in seven circum-Mediterranean areas. Int J Food Sci Nutr. 2008;5: 383-414. https://doi.org/10.1080/09637480701566495.

15. Zhang WJ, Jiang FB, Ou JF. Global pesticide consumption and pollution: with China as a focus. Proc Int Acad Ecol Environ Sci. 2011;2:125 http://www. biological-control.org/chemicalpest/Global-pesticide-consumption-pollution.pdf

16. Lulekal E, Asfaw Z, Kelbessa E, Damme PV. Wild edible plants in Ethiopia: a review on their potential to combat food insecurity. Afr Foc. 2011;24:71-121.

17. Geng YF, Zhang Y, Zhang LL, Wang YH. Research development of food plant ethnobotany-bibliometric and mapping knowledge domains analysis based on web of science. Plant Divers Resour. 2015:4:479-90. https://doi.org/10.7677/ynzwyj201514155.

18. Huang WJ, Long CL. Preliminary ethnobotanical study of wild edible plants in Ledong, Hainan Island, China. Nat Prod Res Dev. 2014;26:273-8.

19. Uprety Y, Poudel RC, Shrestha KK, Rajbhandary S, Tiwari NN, Shrestha UB, Asselin $H$. Diversity of use and local knowledge of wild edible plant resources in Nepal. J Ethnobiol Ethnomed. 2011;1:16. https://doi.org/10.1186/1746-4269-8-16.

20. Pei SJ. Traditional culture and biodiversity conservation. Bull Chin Acad Sci 2011; 2: 190-196. primary: https://doi.org/10.3969/j.issn.1000-3045.2011.02.009.

21. Zheng Y, Fei L, Lu H, Zhi C, Min QW, Bai YY, Liu MC, Cheng SK, Li WH, Fuller AM. Exploring the state of retention of traditional ecological knowledge (TEK) in a Hani rice terrace village, Southwest China. Sustainability. 2014;7: 4497-513. https://doi.org/10.3390/su6074497.
22. Ren HL, Cui BS, Bai JH, Dong SK, Hu B. Distribution of heavy metal in paddy soil of Hani terrace core zone and assessment on its potential ecological risk. Acta Ecol Sin. 2008:4:1625-34. https://doi.org/10.3321/j.issn:1000-0933.2008.04.033.

23. Turner NJ, Łuczaj ŁJ, Migliorini P, Pieroni A, Dreon AL, Sacchetti LE, Paoletti MG. Edible and tended wild plants, traditional ecological knowledge and agroecology. Crit Rev Plant Sci. 2011;1-2:198-225. https://doi.org/10.1080/ 07352689.2011 .554492

24. Kasper-Pakosz R, Pietras M, Łuczaj ŁJ. Wild and native plants and mushrooms sold in the open-air markets of south-eastern Poland. J Ethnobiol Ethnomed. 2016;12:45. https://doi.org/10.1186/s13002-016-0117-8.

25. Alexiades MN, Sheldon JW. Selected guidelines for ethnobotanical research: a field manual. New York: New York Botanical Garden; 1996.

26. Hong LY, Zhuo JX, Lei QY, Zhou JJ, Ahmed S, Wang CY, Long YX, Li FF, Long CL. Ethnobotany of wild plants used for starting fermented beverages in Shui communities of Southwest China. J Ethnobiol Ethnomed. 2015;1:42. https://doi.org/10.1186/s13002-015-0028-0.

27. Pei SJ, Long CL. Applied ethnobotany. Kunming, Yunnan, China: the Nationalities Publishing House of Yunnan; 1998.

28. Ong HG, Ling SM, Win TTM, Kang DH, Lee JH, Kim YD. Ethnobotany of wild medicinal plants used by the Müün ethnic people: a quantitative survey in southern Chin state, Myanmar. J Herb Med. 2017;13:91-6. https://doi.org/10. 1016/j.hermed.2017.09.006.

29. Sujarwo W, Caneva G. Using quantitative indices to evaluate the cultural importance of food and nutraceutical plants: comparative data from the island of Bali (Indonesia). J Cult Herit. 2016;18:342-8. https://doi.org/10.1016/j.culher.2015.06.006.

30. Rop O, Mlcek J, Jurikova T, Neugebauerova J, Vabkova J. Edible flowers-a new promising source of mineral elements in human nutrition. Molecules. 2012;6:6672-83. https://doi.org/10.3390/molecules17066672.

31. Mlcek J, Rop O. Fresh edible flowers of ornamental plants-a new source of nutraceutical foods. Trends Food Sci Technol. 2011;10:561-9. https://doi.org/ 10.1016/j.tifs.2011.04.006.

32. Liu YT, Long CL. Ethnobotanical studies on the edible flowers in Lahu societies. Guihaia. 2007;2:203-10. https://doi.org/10.3969/j.issn.1000-3142.2007.02.013.

33. Xian Y, Guo JX. Assessment study on nutritive compositions and value of main wild vegetables in South China. Food Sci. 2002. https://doi.org/10. 3969/j.issn.1000-3142.2007.02.013

34. Yao J, Gu WL, Hu XW, Guo JC, Fu SP. Progress of Capparis masaikai Lévl and sweet protein Mabinlinll. Chin Agric Sci Bull. 2013;29:1-4.

35. Yang SK, Yang YL, Yang WL. Advances in research and development of Phyllanthus emblica L. Chin J Appl Environ Biol. 2008;6:22. https://doi.org/10. 3724/SP.J.1145.2008.00846.

36. Shan CY, Ren HR, He HL, Zhang WM. Study on antioxidant effective of polysaccharides from Dioscarea subcalva Prain et Burk. Food Ferment Ind. 2009;35:86-9.

37. He HL, Zhang WM, Zhang J, Shan CY, Jiang HF. Comparative studies on rheologies of polysaccharide in Dioscorea subcalva Prain et Burk. and common used plant gums. Nat Prod Res Dev. 2007;5:874-7. https://doi.org/ 10.3969/j.issn.1001-6880.2007.05.035.

38. Kong DG, Zhao Y, Li GH, Chen BJ, Wang XN, Zhou HL, Lou HX, Ren DM, Shen T. The genus Litsea in traditional Chinese medicine: an ethnomedical, phytochemical and pharmacological review. J Ethnopharmacol. 2015;164: 256-64. https://doi.org/10.1016/j.jep.2015.02.020.

39. Fu J, Dai L, Lin Z, Lu H. Houttuynia cordata Thunb: a review of phytochemistry and pharmacology and quality control. Chin Med. 2013;3: 101. https://doi.org/10.4236/cm.2013.43015.

40. Lau KM, Lee KM, Koon CM, Cheung CSF, Lau CP, Ho HM, Lee MYH, Au SWN, Cheng CHK, Lau CBS. Immunomodulatory and anti-SARS activities of Houttuynia cordata. J Ethnopharmacol. 2008;1:79-85. https://doi.org/10.1016/j.jep.2008.03.018.

41. Liu YJ, Fu WG, Cai ZP, Long CL. Analysis of nutritive components and heavy metals in Colocasia gigantea. Food Res Dev. 2016;21:119-22. https://doi.org/ 10.3969/j.issn.1005-6521.2016.21.027.

42. Higgins $C$. The role of traditional ecological knowledge in managing for biodiversity. For Chron. 1998:3:323-6 https://pubs.cif-ifc.org/doi/pdf/10.5558/tfc74323-3.

43. Solomon J, Mishra BP, Venugopal N, Kharlukhi L, Laloo RC. Traditional knowledge and biodiversity conservation in the sacred groves of Meghalaya. Indian J Tradit Know. 2006:4:563-8 http://nopr.niscair.res.in/handle/123456789/6952.

\section{Publisher's Note}

Springer Nature remains neutral with regard to jurisdictional claims in published maps and institutional affiliations. 